\title{
Effect of Schmidt number on the velocity-scalar cospectrum in isotropic turbulence with a mean scalar gradient
}

\author{
By P. A. O’GORMAN AND D. I. PULLIN \\ Graduate Aeronautical Laboratories 105-50, California Institute of Technology, \\ Pasadena, CA 91125, USA
}

(Received 29 June 2004 and in revised form 14 December 2004)

We consider transport of a passive scalar by an isotropic turbulent velocity field in the presence of a mean scalar gradient. The velocity-scalar cospectrum measures the distribution of the mean scalar flux across scales. An inequality is shown to bound the magnitude of the cospectrum in terms of the shell-summed energy and scalar spectra. At high Schmidt number, this bound limits the possible contribution of the sub-Kolmogorov scales to the scalar flux. At low Schmidt number, we derive an asymptotic result for the cospectrum in the inertial-diffusive range, with $\mathrm{a}-11 / 3$ power law wavenumber dependence, and a comparison is made with results from large-eddy simulation. The sparse direct-interaction perturbation (SDIP) is used to calculate the cospectrum for a range of Schmidt numbers. The Lumley scaling result is recovered in the inertial-convective range and the constant of proportionality was calculated. At high Schmidt numbers, the cospectrum is found to decay exponentially in the viscous-convective range, and at low Schmidt numbers, the $-11 / 3$ power law is observed in the inertial-diffusive range. Results are reported for the cospectrum from a direct numerical simulation at a Taylor Reynolds number of 265, and a comparison is made at Schmidt number order unity between theory, simulation and experiment.

\section{Introduction}

The problem of turbulent mixing of a passive scalar in the presence of a mean scalar gradient has been the subject of extensive study, with recent theoretical work focusing on anomalous scaling of the scalar at small scales (Shraiman \& Siggia 2000). As discussed in the review by Warhaft (2000), a number of open issues remain for behaviour of passive scalar statistics in general, many of them related to the issue of local isotropy. The reason for the ubiquity of a mean scalar gradient in studies of passive scalar mixing is that the mean gradient acts as a source of scalar variance, allowing a statistical steady state to be reached. The mean gradient makes the scalar field non-isotropic, and so a mean scalar flux arises. The velocity-scalar cospectrum measures how this flux is distributed across wavenumbers. If, as is thought, the cospectrum decays faster than the scalar or energy spectra, then this is a measure of the approach to isotropy at the smaller scales. Calculation of the scalar flux is of practical importance, and the contribution to the flux from small scales is of relevance to subgrid modelling and large-eddy simulation (Pullin 2000). Also of interest is the effect of the Schmidt number, $S c$, on the cospectrum, and hence the scalar flux, where $S c$ is defined as the ratio of viscosity to the scalar diffusivity. The effect of $S c$ on 
scalar mixing remains a subject of ongoing research, see, for example, the experimental work of Miller \& Dimotakis (1996), and the simulations of Yeung, Xu \& Sreenivasan (2002).

The shell-summed velocity-scalar cospectrum, $C(k)$, is defined so that the mean scalar flux is given by

$$
\overline{u_{1} \theta}=\int_{0}^{\infty} \mathrm{d} k C(k) .
$$

where $\theta$ is the scalar fluctuation, $u_{1}$ is the component of velocity in the direction of the mean scalar gradient, and $k$ is the wavenumber. Shell-summed spectra are sometimes referred to as three-dimensional spectra and are calculated using an integration over a spherical shell in wavenumber space. Lumley (1967) used a similarity hypothesis to predict the shell-summed cospectrum of the velocity and potential temperature. For the case of passive scalar mixing, Lumley's equation (12) for the cospectrum in the inertial-convective range becomes

$$
C(k) \sim \mu \epsilon^{1 / 3} k^{-7 / 3},
$$

where $\mu$ is the mean scalar gradient, and $\epsilon$ is the energy dissipation rate. Mydlarski \& Warhaft (1998) measured the one-dimensional velocity-temperature cospectrum in a wind tunnel under conditions for which the temperature was a passive scalar. They found a wavenumber dependence of approximately $k^{-2}$ in the inertial-convective range for a Taylor Reynolds numbers, $R_{\lambda}$, of 582; see also Mydlarski (2003). Bos et al. (2004) studied the importance of the pressure term in the evolution equation for the cospectrum and found a $k^{-2}$ scaling range for the cospectrum using large-eddy simulation (LES). Herr, Wang \& Collins (1996) performed an EDQNM calculation of the cospectrum and compared it with direct numerical simulation (DNS) at an $R_{\lambda}$ of 81, although it should be noted that two constants were chosen in the EDQNM calculation by matching the EDQNM and DNS cospectra. There has also been work by Kaneda \& Yoshida (2004) and Gargett, Merryfield \& Holloway (2003) on the related problem of the buoyancy flux spectrum in stably stratified turbulence, although here we will exclusively consider the case of a passive scalar.

The velocity-scalar cospectrum was studied by O'Gorman \& Pullin (2003) using the stretched-spiral vortex model introduced by Lundgren (1982). They found that the contribution to the cospectrum from the velocity directed parallel to the vortex tube axes had a $k^{-5 / 3}$ wavenumber-dependence to leading order. The next-order term had a $k^{-7 / 3}$ dependence, but its sign depended on the initial conditions. The contribution from the velocity in the plane of the vortex structure depended on the choice of vortex core. In addition, exact relations were derived relating the shell-summed and one-dimensional cospectra, and the quadrature spectrum was shown to be zero.

Here we utilize the sparse direct-interaction perturbation (SDIP) introduced by Kida \& Goto (1997, hereinafter referred to as KG) with the name Lagrangian direct-interaction approximation. It is a renormalized closure theory for second-order turbulent statistics, and applies a similar procedure to Kraichnan's direct-interaction approximation (DIA), (Kraichnan 1959), in a Lagrangian framework. The SDIP is simpler than the Lagrangian history DIA of Kraichnan (1965), and yields the same integro-differential equations as the Lagrangian renormalized approximation (LRA) obtained earlier by Kaneda (1981). This latter closure has been applied to passive scalar mixing in two and three dimensions, see Kaneda (1986), Gotoh (1989) and Gotoh, Nagaki \& Kaneda (2000). The SDIP has been used to calculate the energy spectrum (KG), and the scalar spectrum (Goto \& Kida 1999), and the resulting scaling exponents were found to be in agreement with classical phenomenology. 
Goto \& Kida (2002) applied the SDIP to a simpler model to understand better the basis of the approximation. In light of the importance of sparse coupling in the approximation, the name sparse direct-interaction perturbation was then chosen in place of Lagrangian direct-interaction approximation. There are other two-point closures that have been successfully used to calculate turbulent energy and scalar spectra, for example, the local energy-transfer (LET) theory of McComb, Filipiak \& Shanmugasundaram (1992). The SDIP is particularly promising for use here since Goto \& Kida (1999) were able to use it to recover a number of classical scaling results for the scalar spectrum at different $S c$.

In this paper we study the velocity-scalar cospectrum using a combination of theory and simulation. The cospectrum is defined in $\S 2$, and the form of the cospectrum at different $S c$ is considered in $\S 3$. An inequality is derived that bounds the magnitude of the cospectrum, and this is shown to have implications for the cospectrum at high $S c$. This inequality is an extension of the one-dimensional cross-spectrum inequality to the shell-summed case, and applied in particular to the velocity-scalar cospectrum. At low $S c$, the asymptotic form of the cospectrum in the inertial-diffusive range is derived. The derivation is similar to the argument of Batchelor, Howells \& Townsend (1959) for the form of the scalar spectrum in the inertial-diffusive range. The use of the SDIP closure to calculate the cospectrum is described in $\S 4$. The Lumley (1967) form is recovered in the inertial-convective range, and the closed equations are solved numerically for a range of $S c$. Finally, in $\S 5$, we report results for the cospectrum from both DNS and LES. The DNS was performed at $R_{\lambda}$ of 265 and $S c$ of 0.7 , and a comparison is made with theory and experiment at this $S c$. The LES was performed at low $S c$ and agreement is found with the asymptotic form for the cospectrum in the inertial-diffusive range.

\section{The velocity-scalar cospectrum}

We consider a passive scalar mixed by an incompressible, statistically homogeneous and isotropic velocity field, $u_{i}(\boldsymbol{x}, t)$. The scalar is assumed to have a uniform mean scalar gradient, $\mu$, in the 1 direction, so that we can decompose the scalar as $\mu x_{1}+\theta(\boldsymbol{x}, t)$. The scalar fluctuation $\theta(\boldsymbol{x}, t)$ is statistically homogeneous, and axisymmetric about the $x_{1}$ axis, but not isotropic. By definition it has zero mean, $\overline{\theta(\boldsymbol{x}, t)}=0$, where the overbar indicates an ensemble average.

If we define the velocity-scalar correlation by

$$
\mathscr{R}_{u_{i} \theta}(\boldsymbol{r})=\overline{u_{i}(\boldsymbol{x}, t) \theta(\boldsymbol{x}+\boldsymbol{r}, t)},
$$

then the shell-summed cospectrum of the scalar and the velocity component $u_{1}$ is defined by

$$
C(k)=\frac{1}{(2 \pi)^{3}} \int \mathrm{d} S_{k} \int \mathrm{d} \boldsymbol{r} \mathscr{R}_{u_{1} \theta}(\boldsymbol{r}) \exp (-\mathrm{i} \boldsymbol{k} \cdot \boldsymbol{r}) .
$$

Here the $\int \mathrm{d} S_{k}$ integral is a surface integral over a spherical shell in wavenumber space, and may be written as $k^{2} \int_{0}^{\pi} \mathrm{d} \psi_{k} \int_{0}^{2 \pi} \mathrm{d} \phi_{k} \sin \psi_{k}$. The $\int \mathrm{d} \boldsymbol{r}$ integral is a volume integral over all space. The shell-summed cospectrum has no imaginary part, as may be seen by performing the shell integral of $\exp (-\mathrm{i} \boldsymbol{k} \cdot \boldsymbol{r})$. An important property of the cospectrum is that it integrates to the scalar flux, as given by (1.1). The shell-summed cospectrum is thus a measure of the distribution of the scalar flux across scales.

One-dimensional spectra are often more convenient for experimental measurement, and so we also define a one-dimensional velocity-scalar cospectrum,

$$
C^{1 d}\left(k_{3}\right)=\frac{1}{\pi} \int_{-\infty}^{\infty} \mathscr{R}_{u_{1} \theta}\left(0,0, r_{3}\right) \cos \left(k_{3} r_{3}\right) \mathrm{d} r_{3},
$$


see Bendat \& Piersol (1986). The one-dimensional cospectrum also integrates to the scalar flux,

$$
\overline{u_{1} \theta}=\int_{0}^{\infty} C^{1 d}\left(k_{3}\right) \mathrm{d} k_{3} .
$$

It was shown in O'Gorman \& Pullin (2003) that only the cospectrum of the scalar and the velocity component in the direction of the mean scalar gradient is non-zero, where this holds for both the shell-summed and one-dimensional cospectra.

\section{The cospectrum at small and large Schmidt number}

We consider the effects of Schmidt number on the cospectrum, where the Schmidt number is defined as the ratio of viscosity to scalar diffusivity, $S_{c}=v / \kappa$. In later sections we will calculate the cospectrum using turbulence theory and DNS, but we can use some simpler analysis to limit the possible behaviour of the cospectrum, and in the case of low $S c$ to predict its asymptotic form.

\subsection{The cospectrum inequality}

Here we will derive an upper bound for the magnitude of the shell-summed velocityscalar cospectrum in terms of the energy and scalar spectra. This bound has close ties to the one-dimensional cross-spectrum inequality and coherence function, discussed in Bendat \& Piersol (1986). In effect, we are extending the one-dimensional crossspectrum inequality to the three-dimensional shell-summed case, and applying it in particular to the velocity-scalar cospectrum.

It is convenient to use the formulation of the SDIP calculation of $\S 4$, where we first work in a periodic box of side $L$, and then take the limit $L \rightarrow \infty$. The velocity field $u_{i}(\boldsymbol{x}, t)$ can be decomposed as

$$
u_{i}(\boldsymbol{x}, t)=\left(\frac{2 \pi}{L}\right)^{3} \sum_{\boldsymbol{k}} \tilde{u}_{i}(\boldsymbol{k}, t) \exp (\mathrm{i} \boldsymbol{k} \cdot \boldsymbol{x}),
$$

where $k_{i}=2 \pi n_{i} / L$, and $n_{i} \in \mathbb{Z}$. The inverse Fourier transform is given by

$$
\tilde{u}_{i}(\boldsymbol{k}, t)=\left(\frac{1}{2 \pi}\right)^{3} \int \mathrm{d}^{3} \boldsymbol{x} u_{i}(\boldsymbol{x}, t) \exp (-\mathrm{i} \boldsymbol{k} \cdot \boldsymbol{x}),
$$

and a similar transformation is defined for the scalar fluctuation, $\theta(\boldsymbol{x}, t)$. We then define the second-order statistical quantities

$$
\begin{aligned}
\tilde{V}_{i j}(\boldsymbol{k}, t, t) & =\left(\frac{2 \pi}{L}\right)^{3} \overline{\tilde{u}_{i}(\boldsymbol{k}, t) \tilde{u}_{j}(-\boldsymbol{k}, t),} \\
\tilde{Z}(\boldsymbol{k}, t, t) & =\left(\frac{2 \pi}{L}\right)^{3} \overline{\tilde{\theta}(\boldsymbol{k}, t) \tilde{\theta}(-\boldsymbol{k}, t),} \\
\tilde{W}_{i}(\boldsymbol{k}, t, t) & =\left(\frac{2 \pi}{L}\right)^{3} \overline{\tilde{\theta}(\boldsymbol{k}, t) \tilde{u}_{i}(-\boldsymbol{k}, t) .}
\end{aligned}
$$

The double reference to the time $t$ is included to be consistent with the definition of more complicated Lagrangian quantities in $\S 4$.

For a given instance in the ensemble, we have

$$
\operatorname{Re}\left(\tilde{\theta}(\boldsymbol{k}, t) \tilde{u}_{1}(-\boldsymbol{k}, t)\right) \leqslant|\tilde{\theta}(\boldsymbol{k}, t)|\left|\tilde{u}_{1}(\boldsymbol{k}, t)\right|,
$$


where we have used $\tilde{u}(-\boldsymbol{k}, t)=\tilde{u}(\boldsymbol{k}, t)^{*}$. Taking an ensemble average we find that

$$
\operatorname{Re}\left(\overline{\tilde{\theta}(\boldsymbol{k}, t) \tilde{u}_{1}(-\boldsymbol{k}, t)}\right) \leqslant \overline{|\tilde{\theta}(\boldsymbol{k}, t)|\left|\tilde{u}_{1}(\boldsymbol{k}, t)\right|} \leqslant\left(\overline{|\tilde{\theta}(\boldsymbol{k}, t)|^{2}} \overline{\left|\tilde{u}_{1}(\boldsymbol{k}, t)\right|^{2}}\right)^{1 / 2} .
$$

The second inequality can be derived by considering the expression

$$
\overline{\left(|\tilde{\theta}(\boldsymbol{k}, t)|+\xi\left|\tilde{u}_{1}(\boldsymbol{k}, t)\right|\right)^{2}},
$$

as a quadratic in the real number $\xi$, and requiring that it be non-negative.

Taking the limit $L \rightarrow \infty$, we can relate $\tilde{V}_{i j}(\boldsymbol{k}, t, t), \tilde{Z}(\boldsymbol{k}, t, t)$ and $\tilde{W}_{i}(\boldsymbol{k}, t, t)$ to power spectral density functions. The shell-summed energy spectrum, $E(k)$, scalar spectrum, $\Theta(k)$, and velocity-scalar cospectrum, $C(k)$, are given by

$$
\begin{aligned}
& E(k)=\frac{1}{2} \int \mathrm{d} S_{k} \tilde{V}_{i i}(\boldsymbol{k}, t, t), \\
& \Theta(k)=\int \mathrm{d} S_{k} \tilde{Z}(\boldsymbol{k}, t, t), \\
& C(k)=\int \mathrm{d} S_{k} \tilde{W}_{1}(\boldsymbol{k}, t, t),
\end{aligned}
$$

where $\int \mathrm{d} S_{k}$ again denotes a surface integral over a shell in wavenumber space. We will now use the fact that the shell-summed cospectrum has no imaginary part. Noting that the isotropy of the velocity field implies that $\tilde{V}_{11}(\boldsymbol{k}, t, t)=E(k) /\left(6 \pi k^{2}\right)$, and using inequality (3.7), we deduce that

$$
C(k) \leqslant \frac{1}{k}\left(\int \mathrm{d} S_{k} \tilde{Z}(\boldsymbol{k}, t, t)^{1 / 2}\right)\left(\frac{E(k)}{6 \pi}\right)^{1 / 2} .
$$

Similarly we can show that inequality (3.12) holds for $-C(k)$, and so it also holds for the magnitude $|C(k)|$. The scalar spectrum is anisotropic, and so we cannot perform the surface integral without further knowledge of $\tilde{Z}(\boldsymbol{k}, t, t)$. Nonetheless, we can find a bound in terms of the shell-summed scalar spectrum. Using the Cauchy-Schwartz inequality we have

$$
\int \mathrm{d} S_{k} \tilde{Z}(\boldsymbol{k}, t, t)^{1 / 2} 1^{1 / 2} \leqslant\left(\int \mathrm{d} S_{k} \tilde{Z}(\boldsymbol{k}, t, t)\right)^{1 / 2}\left(\int \mathrm{d} S_{k}\right)^{1 / 2}=\left(4 \pi k^{2} \Theta(k)\right)^{1 / 2},
$$

and so,

$$
|C(k)| \leqslant\left(\frac{2 E(k) \Theta(k)}{3}\right)^{1 / 2} .
$$

A tighter bound might be deduced with more detailed knowledge of the scalar anisotropy.

\subsection{Implications of the bound}

From (3.14) we see that the magnitude of the cospectrum is bounded by the geometric mean of the scalar and energy spectra multiplied by a constant of order unity. To discuss the implications of this, we first briefly review the phenomenology of the scalar spectrum at different $S c$.

We assume that the Reynolds number is sufficiently large for an inertialconvective range to exist. We define $k_{P}$ as the wavenumber at the peak of the energy spectrum or the scalar spectrum, whichever wavenumber is greater. The Kolmogorov wavenumber is defined by $k_{K}=\left(\epsilon / v^{3}\right)^{1 / 4}$, the Batchelor wavenumber is given by $k_{B}=\left(\epsilon / v \kappa^{2}\right)^{1 / 4}=S_{c}^{1 / 2} k_{K}$, and the Obukhov-Corrsin wavenumber is given by 
$k_{C}=\left(\epsilon / \kappa^{3}\right)^{1 / 4}=S_{c}^{3 / 4} k_{K}$. Then, for wavenumbers in the inertial-convective range, the scalar spectrum has the form

$$
\Theta(k) \propto \epsilon_{\theta} \epsilon^{-1 / 3} k^{-5 / 3}, \quad k_{P} \ll k \ll \min \left(k_{K}, k_{C}\right),
$$

where $\epsilon_{\theta}$ is the scalar dissipation and the constant of proportionality is known as the Obukhov-Corrsin constant (see Tennekes \& Lumley 1974). For large $S_{c}$ a different power law behavior is thought to exist in the viscous-convective range (Batchelor 1959),

$$
\Theta(k) \propto \epsilon_{\theta} v^{1 / 2} \epsilon^{-1 / 2} k^{-1}, \quad k_{K} \ll k \ll k_{B}, \quad S c \gg 1 .
$$

For very small $S_{c}$, in the inertial-diffusive range,

$$
\Theta(k)=\frac{1}{3} \kappa^{-3} K \epsilon^{2 / 3} \epsilon_{\theta} k^{-17 / 3}\left(1+\frac{2 \kappa \mu^{2}}{\epsilon_{\theta}}\right), \quad k_{C} \ll k \ll k_{K}, \quad S c \ll 1,
$$

where $K$ is the Kolmogorov constant. We show this in Appendix A, using a direct re-derivation of a result of Batchelor et al. (1959), modified for the case of a mean scalar gradient. As noted by Chasnov (1991), the effect of the mean scalar gradient can be captured by replacing the scalar dissipation with $\epsilon_{\theta}+2 \kappa \mu^{2}$ in the Batchelor et al. (1959) result. There are other theoretical predictions for the scalar spectrum in the inertial-diffusive range; for example, Gibson (1968) found a $k^{-3}$ wavenumber dependence. Finally, in the viscous-diffusive range, the scalar spectrum decays exponentially.

Now consider the velocity-scalar cospectrum for the case of large $S c$ in the viscousconvective range. According to (3.16), the scalar spectrum has a $k^{-1}$ wavenumber dependence, whereas the energy spectrum will be decaying exponentially with wavenumber because $k \gg k_{K}$. Therefore, the bound given by inequality (3.14) will decay exponentially in this range, and we expect that the cospectrum will also decay exponentially. This would imply that the contribution to the mean scalar flux at length scales smaller than the Kolmogorov lengthscale is very small, even if $S_{c}$ is very large. It should be noted that if the scaling law given by (3.16) is correct, then as $S_{c} \rightarrow \infty$ the scalar variance is unbounded; see Dimotakis \& Miller (1990) for a discussion of this issue.

In contrast, for the case of small $S_{c}$ in the inertial-diffusive range, the scalar spectrum has a wavenumber dependence of $k^{-17 / 3}$ according to (3.17), the energy spectrum has a $k^{-5 / 3}$ wavenumber dependence because $k_{P} \ll k \ll k_{K}$, and so the bound given by inequality (3.14) has a $k^{-11 / 3}$ dependence. Therefore, in this wavenumber range we cannot exclude either exponential or power law behaviour of the cospectrum based on the inequality alone. However, we will be able to derive an asymptotic form for the inertial-diffusive range in the next subsection.

\subsection{Asymptotic form in the inertial-diffusive range}

We consider the case of low $S_{c}$, and wavenumbers in the inertial-diffusive range, $k_{C} \ll k \ll k_{K}$. The advection-diffusion equation for the scalar fluctuation is given by

$$
\frac{\partial}{\partial t} \theta(\boldsymbol{x}, t)+u_{j}(\boldsymbol{x}, t) \frac{\partial}{\partial x_{j}} \theta(\boldsymbol{x}, t)=\kappa \frac{\partial^{2}}{\partial x_{j} \partial x_{j}} \theta(\boldsymbol{x}, t)-\mu u_{1}(\boldsymbol{x}, t),
$$

where we note the gradient forcing term. This can be written in Fourier space as

$$
\left[\frac{\partial}{\partial t}+\kappa k^{2}\right] \tilde{\theta}(\boldsymbol{k}, t)=-\mu \tilde{u}_{1}(\boldsymbol{k}, t)-\mathrm{i}\left(\frac{2 \pi}{L}\right)^{3} \sum_{q} q_{j} \tilde{u}_{j}(\boldsymbol{k}-\boldsymbol{q}, t) \tilde{\theta}(\boldsymbol{q}, t) .
$$


Following the argument of Batchelor et al. (1959), we note that the convolution sum in (3.19) is dominated by wavenumbers $\boldsymbol{q}$ smaller than $k_{C}$, that is $|\boldsymbol{q}|<k_{C}$. This is justified because the scalar spectrum drops off rapidly for higher wavenumbers. Then, assuming that $k \gg k_{C}$, implies that $|\boldsymbol{k}-\boldsymbol{q}| \simeq k$. We now argue that the time scales of $\tilde{u}_{1}(\boldsymbol{k}, t), \tilde{u}_{j}(\boldsymbol{k}-\boldsymbol{q}, t)$ and $\tilde{\theta}(\boldsymbol{q}, t)$ are much longer that that of $\tilde{\theta}(\boldsymbol{k}, t)$. This can be seen in the case of $\tilde{u}_{1}(\boldsymbol{k}, t)$ and $\tilde{u}_{j}(\boldsymbol{k}-\boldsymbol{q}, t)$ by comparing the inertial time scale $\epsilon^{-1 / 3} k^{-2 / 3}$ with the diffusive time scale $\kappa^{-1} k^{-2}$ and using $k \gg k_{C}$. Therefore, we can view the right-hand side of (3.19) as a quasi-stationary source term and make a stationary balance approximation by neglecting the time derivative. Multiplying by $u_{1}(-\boldsymbol{k}, t)$, taking an ensemble average, and using the definition (3.5) of $\tilde{W}_{i}(\boldsymbol{k}, t, t)$ we find

$$
\begin{aligned}
\tilde{W}_{1}(\boldsymbol{k}, t, t)=-\frac{\mu}{\kappa k^{2}}\left(\frac{2 \pi}{L}\right)^{3} & \overline{\tilde{u}_{1}(-\boldsymbol{k}, t) \tilde{u}_{1}(\boldsymbol{k}, t)} \\
& -\frac{\mathrm{i}}{\kappa k^{2}}\left(\frac{2 \pi}{L}\right)^{6} \sum_{q} q_{j} \overline{\tilde{u}_{j}(\boldsymbol{k}-\boldsymbol{q}, t) \tilde{\theta}(\boldsymbol{q}, t) \tilde{u}_{1}(-\boldsymbol{k}, t) .} .
\end{aligned}
$$

We have already argued that $|\boldsymbol{q}| \ll|\boldsymbol{k}-\boldsymbol{q}| \simeq k$, and so we make the further approximation that $\tilde{\theta}(\boldsymbol{q}, t)$ is statistically independent of $\tilde{u}_{j}(\boldsymbol{k}-\boldsymbol{q}, t)$ and $\tilde{u}_{1}(-\boldsymbol{k}, t)$. The mean $\overline{\tilde{\theta}(\boldsymbol{q}, t)}$ is zero, and so

$$
\tilde{W}_{1}(\boldsymbol{k}, t, t)=-\frac{\mu}{\kappa k^{2}} \tilde{V}_{11}(\boldsymbol{k}, t, t) .
$$

Taking the limit $L \rightarrow \infty$, and using (3.9) and (3.11) to make contact with shell-summed spectra we find

$$
C(k)=-\frac{2 \mu}{3 \kappa k^{2}} E(k) .
$$

The wavenumber $k$ is in the inertial range, and so

$$
E(k)=K \epsilon^{2 / 3} k^{-5 / 3},
$$

where $K$ is the Kolmogorov constant, with the result

$$
C(k)=-\frac{2 \mu K}{3 \kappa} k^{-11 / 3} \epsilon^{2 / 3} .
$$

Thus the cospectrum has a $k^{-11 / 3}$ power law wavenumber-dependence in the inertialdiffusive range. The asymptotic form (3.24) is found to be in agreement with the SDIP result in $\S 4.4$, and with results from LES in $\S 5.3$.

We can also compare our asymptotic result for the cospectrum in the inertialdiffusive range with the bound given by the cospectrum inequality. Substituting (3.23) and (3.17) into (3.14) we find

$$
|C(k)| \leqslant \frac{2}{3} \mu K \epsilon^{2 / 3} k^{-11 / 3} \kappa^{-1}\left(\frac{\epsilon_{\theta}}{2 \kappa \mu^{2}}+1\right)^{1 / 2} .
$$

Thus the bound exceeds the magnitude of our asymptotic result for the cospectrum by a factor $\left(\epsilon_{\theta} /\left(2 \kappa \mu^{2}\right)+1\right)^{1 / 2}$.

In summary, we have used some simple analyses to characterize the behaviour of the cospectrum at both large and small $S c$. We have mainly discussed inequality (3.14) in the context of the viscous-convective range at large $S c$, but it also applies more generally. To learn more about the cospectrum in the inertial-convective range, and for a more detailed characterization of the cospectrum at all scales we turn now to the SDIP and numerical simulation. 


\section{SDIP calculation}

In this section, we will discuss the use of the SDIP to calculate the velocity-scalar cospectrum for a range of $S_{c}$. We emphasize that we consider a statistically isotropic velocity field, but a statistically non-isotropic scalar field. Fortunately, we will be able to use incompressibility of the velocity field and the statistical axisymmetry of the scalar field to describe the cospectrum using a single isotropic function. The resulting SDIP equations are still considerably more complicated than those derived for the scalar spectrum in the isotropic case (Goto \& Kida 1999).

The basic formulation is described in $\S 4.1$. The SDIP is applied in Appendix B and the resulting equations are summarized in $\S 4.2$. In $\S 4.3$, we solve the equations in the inertial-convective range, and in $\S 4.4$ we find the asymptotic solution in the limit of low $S_{c}$. Lastly, in $\S 4.5$, we solve the equations numerically for a range of $S_{c}$.

\subsection{Basic formulation}

The notation we use is consistent with that of Goto \& Kida (1999). A more detailed account of some of the basic equations can be found in Section II of that paper, although it should be noted that they deal with a statistically isotropic scalar field without a mean gradient.

The velocity field evolves according to the Navier-Stokes equations,

$$
\frac{\partial}{\partial t} u_{i}(\boldsymbol{x}, t)+u_{j}(\boldsymbol{x}, t) \frac{\partial}{\partial x_{j}} u_{i}(\boldsymbol{x}, t)=-\frac{\partial}{\partial x_{i}} \pi(\boldsymbol{x}, t)+v \frac{\partial^{2}}{\partial x_{j} \partial x_{j}} u_{i}(\boldsymbol{x}, t),
$$

where $\pi(x, t)$ is the pressure-density ratio, and the continuity condition,

$$
\frac{\partial}{\partial x_{i}} u_{i}(\boldsymbol{x}, t)=0 \text {. }
$$

The scalar fluctuation evolves according to the advection-diffusion equation given by (3.18). It is convenient to make use of a Lagrangian position function, $\psi\left(\boldsymbol{x}, t \mid \boldsymbol{x}^{\prime}, t^{\prime}\right)$, defined by

$$
\psi\left(\boldsymbol{x}, t \mid \boldsymbol{x}^{\prime}, t^{\prime}\right)=\delta^{3}\left(\boldsymbol{x}-\boldsymbol{y}\left(t \mid \boldsymbol{x}^{\prime}, t^{\prime}\right)\right),
$$

where a fluid element that is located at $\boldsymbol{x}^{\prime}$ at time $t^{\prime}$, is located at $\boldsymbol{y}\left(t \mid \boldsymbol{x}^{\prime}, t^{\prime}\right)$ at time $t \geqslant t^{\prime}$. The Lagrangian position function evolves according to

$$
\frac{\partial}{\partial t} \psi\left(\boldsymbol{x}, t \mid \boldsymbol{x}^{\prime}, t^{\prime}\right)+u_{j}(\boldsymbol{x}, t) \frac{\partial}{\partial x_{j}} \psi\left(\boldsymbol{x}, t \mid \boldsymbol{x}^{\prime}, t^{\prime}\right)=0
$$

with initial condition

$$
\psi\left(\boldsymbol{x}, t \mid \boldsymbol{x}^{\prime}, t\right)=\delta^{3}\left(\boldsymbol{x}-\boldsymbol{x}^{\prime}\right) .
$$

We can then define Lagrangian velocity and scalar fields as

$$
v_{i}\left(t \mid \boldsymbol{x}, t^{\prime}\right)=\int \mathrm{d}^{3} \boldsymbol{x}^{\prime} u_{i}\left(\boldsymbol{x}^{\prime}, t\right) \psi\left(\boldsymbol{x}^{\prime}, t \mid \boldsymbol{x}, t^{\prime}\right)
$$

and

$$
\theta^{(L)}\left(t \mid \boldsymbol{x}, t^{\prime}\right)=\int \mathrm{d}^{3} \boldsymbol{x}^{\prime} \theta\left(\boldsymbol{x}^{\prime}, t\right) \psi\left(\boldsymbol{x}^{\prime}, t \mid \boldsymbol{x}, t^{\prime}\right) .
$$

The Lagrangian velocity autocorrelation function is given by

$$
V_{i j}\left(\boldsymbol{r}, t, t^{\prime}\right)=\overline{v_{i}\left(t \mid \boldsymbol{x}+\boldsymbol{r}, t^{\prime}\right) u_{j}\left(\boldsymbol{x}, t^{\prime}\right)}
$$


We wish to calculate the velocity-scalar cospectrum, and so define Lagrangian crosscorrelation functions,

$$
W_{i}\left(\boldsymbol{r}, t, t^{\prime}\right)=\overline{\theta^{(L)}\left(t \mid \boldsymbol{x}+\boldsymbol{r}, t^{\prime}\right) u_{i}\left(\boldsymbol{x}, t^{\prime}\right)}
$$

and

$$
Y_{i}\left(\boldsymbol{r}, t, t^{\prime}\right)=\overline{v_{i}\left(t \mid \boldsymbol{x}+\boldsymbol{r}, t^{\prime}\right) \theta\left(\boldsymbol{x}, t^{\prime}\right)}
$$

The SDIP is formulated in Fourier space by first assuming that the flow is in a periodic box of side $L$. The velocity field $u_{i}(\boldsymbol{x}, t)$ can then be decomposed as in (3.1), and the inverse Fourier transform is given by (3.2). Similar transforms are defined for the other variables. The limit $L \rightarrow \infty$ is taken at a later stage. The Fourier transforms of the two-point statistics are given by

$$
\begin{aligned}
\tilde{V}_{i j}\left(\boldsymbol{k}, t, t^{\prime}\right) & =\left(\frac{2 \pi}{L}\right)^{3} \overline{\tilde{v}_{i}\left(t \mid \boldsymbol{k}, t^{\prime}\right) \tilde{u}_{j}\left(-\boldsymbol{k}, t^{\prime}\right),} \\
\tilde{W}_{i}\left(\boldsymbol{k}, t, t^{\prime}\right) & =\left(\frac{2 \pi}{L}\right)^{3} \overline{\tilde{\theta}^{(L)}\left(t \mid \boldsymbol{k}, t^{\prime}\right) \tilde{u}_{i}\left(-\boldsymbol{k}, t^{\prime}\right),} \\
\tilde{Y}_{i}\left(\boldsymbol{k}, t, t^{\prime}\right) & =\left(\frac{2 \pi}{L}\right)^{3} \overline{\tilde{v}_{i}\left(t \mid \boldsymbol{k}, t^{\prime}\right) \tilde{\theta}\left(-\boldsymbol{k}, t^{\prime}\right) .}
\end{aligned}
$$

It is often more convenient to work with incompressible projections of $\tilde{V}_{i j}\left(\boldsymbol{k}, t, t^{\prime}\right)$ and $\tilde{Y}_{i}\left(\boldsymbol{k}, t, t^{\prime}\right)$, and so we define

$$
\begin{aligned}
\tilde{Q}_{i j}\left(\boldsymbol{k}, t, t^{\prime}\right) & =\tilde{P}_{i m}(\boldsymbol{k}) \tilde{V}_{m j}\left(\boldsymbol{k}, t, t^{\prime}\right), \\
\tilde{X}_{i}\left(\boldsymbol{k}, t, t^{\prime}\right) & =\tilde{P}_{i m}(\boldsymbol{k}) \tilde{Y}_{m}\left(\boldsymbol{k}, t, t^{\prime}\right),
\end{aligned}
$$

where $\tilde{P}_{i j}(\boldsymbol{k})=\delta_{i j}-k_{i} k_{j} / k^{2}$. After taking the limit $L \rightarrow \infty$ we can relate $\tilde{V}_{i i}(\boldsymbol{k}, t, t)$ to the shell-summed energy spectrum, $E(k)$, and $\tilde{W}_{1}(\boldsymbol{k}, t, t)$ to the shell-summed velocity-scalar cospectrum, $C(k)$, see (3.9) and (3.11), respectively. The shell-summed cospectrum can also be related to $\tilde{Y}$ by noting that $\tilde{W}_{1}(\boldsymbol{k}, t, t)=\tilde{Y}_{1}(-\boldsymbol{k}, t, t)$.

\subsection{Closed equations}

The application of the SDIP closure is given in Appendix B. Here, we will summarize the resulting equations for the case of an isotropic velocity field and a scalar with a mean gradient in the ' 1 ' direction. We also assume statistical stationarity. With these assumptions it is shown in Appendix B that we can write

$$
\tilde{Q}_{i j}\left(\boldsymbol{k}, t, t^{\prime}\right)=\frac{1}{2} \tilde{P}_{i j}(\boldsymbol{k}) Q^{\dagger}\left(k, t-t^{\prime}\right)
$$

and

$$
\tilde{W}_{i}\left(\boldsymbol{k}, t, t^{\prime}\right)=\tilde{P}_{i 1}(\boldsymbol{k}) \mu W^{\dagger}\left(k, t-t^{\prime}\right),
$$

for some functions $Q^{\dagger}\left(k, t-t^{\prime}\right)$ and $W^{\dagger}\left(k, t-t^{\prime}\right)$.

The closed SDIP equations are then given by

$$
W^{\dagger}(k, t)=\left(-\frac{1}{2} \int_{0}^{t} Q^{\dagger}\left(k, t^{\prime}\right) \exp \left[\kappa k^{2} t^{\prime}\right] \mathrm{d} t^{\prime}+W^{\dagger}(k, 0)\right) \exp \left[-\kappa k^{2} t\right]
$$

and

$$
2 k^{2}(v+\kappa) W^{\dagger}(k, 0)+Q^{\dagger}(k, 0)=\frac{1}{2} \iint_{\triangle_{k}} \mathrm{~d} p \mathrm{~d} q \frac{\pi p q}{k} \frac{\sigma(k, p, q)}{q^{2}} \int_{0}^{\infty} \mathrm{d} t M(k, p, q, t),
$$


where

$$
\begin{aligned}
M(k, p, q, t)= & \left(k^{2}-q^{2}\right)\left(k^{2}-p^{2}+q^{2}\right) Q^{\dagger}(k, t) W^{\dagger}(q, t) Q^{\dagger}(p, t) Q^{\dagger}(p, 0)^{-1} \\
& +\left(\left(p^{2}-q^{2}\right)^{2}-k^{2}\left(p^{2}-3 q^{2}\right)\right) Q^{\dagger}(p, t) W^{\dagger}(q, t) Q^{\dagger}(k, t) Q^{\dagger}(k, 0)^{-1} \\
& +\exp \left[-\kappa q^{2} t\right] Q^{\dagger}(k, t) Q^{\dagger}(p, t) q^{2}\left(-4 k^{2} W^{\dagger}(k, 0) Q^{\dagger}(k, 0)^{-1}\right. \\
& \left.-\left(k^{2}+p^{2}-q^{2}\right) W^{\dagger}(p, 0) Q^{\dagger}(p, 0)^{-1}\right)+\left(2 p^{2}\left(q^{2}+k^{2}-p^{2}\right)\right. \\
& \left.-4 q^{2} k^{2}\right) Q^{\dagger}(p, t) W^{\dagger}(k, t) Q^{\dagger}(q, t) Q^{\dagger}(q, 0)^{-1}+\exp \left[-\kappa k^{2} t\right]\left(\left(p^{2}-q^{2}\right)^{2}\right. \\
& \left.-k^{2}\left(p^{2}-3 q^{2}\right)\right) Q^{\dagger}(p, t) W^{\dagger}(q, 0) Q^{\dagger}(q, t) Q^{\dagger}(q, 0)^{-1}
\end{aligned}
$$

and

$$
\sigma(k, p, q)=\frac{(k+p+q)(k+p-q)(k-p+q)(-k+p+q)}{4 p^{2} k^{2}} .
$$

The wavenumber integral is given by

$$
\iint_{\triangle_{k}} \mathrm{~d} p \mathrm{~d} q=\int_{0}^{\infty} \mathrm{d} p \int_{|k-p|}^{k+p} \mathrm{~d} q
$$

The linear integral equation (4.19), together with (4.18), are sufficient to determine $W^{\dagger}(k, 0)$ when $Q^{\dagger}(k, t)$ has been specified.

\subsection{Inertial-convective range}

Here we will solve (4.18) and (4.19) for $W^{\dagger}(k, 0)$ in the inertial-convective range, that is in the wavenumber range where viscosity and diffusivity are unimportant. We introduce a non-dimensional wavenumber $\hat{k}=k / k_{K}$, and a non-dimensional time $\tau=t \epsilon^{1 / 3} k^{2 / 3}$, where $k_{K}=\left(\epsilon / \nu^{3}\right)^{1 / 4}$ is the Kolmogorov wavenumber. It was shown in $\mathrm{KG}$ that according to the SDIP, the velocity correlation function $Q^{\dagger}(k, t)$ can be written as

$$
Q^{\dagger}(k, t)=\frac{1}{2 \pi} K \epsilon^{2 / 3} k^{-11 / 3} Q(\hat{k}, \tau),
$$

where $K=1.722$ is the Kolmogorov constant. In particular, in the inertial range we have

$$
Q^{\dagger}(k, t)=\frac{1}{2 \pi} K \epsilon^{2 / 3} k^{-11 / 3} Q(0, \tau) .
$$

Assuming we are in the inertial-convective range means that we can effectively set $v$ and $\kappa$ to zero in (4.18), (4.19) and (4.20). Thus, there are no characteristic scales, and we can look for solutions of the form

$$
W^{\dagger}(k, 0)=-\frac{1}{2 \pi} \gamma \epsilon^{1 / 3} k^{-13 / 3},
$$

where $\gamma$ is a constant to be determined. Then, by (4.18),

$$
W^{\dagger}(k, t)=W^{\dagger}(k, 0)\left(1+\frac{K}{2 \gamma} R\left(0, k^{2 / 3} \epsilon^{1 / 3} t\right)\right),
$$

where $R(\hat{k}, \tau)$ is given by

$$
R(\hat{k}, \tau)=\exp \left[-\hat{k}^{4 / 3} \tau / S_{c}\right] \int_{0}^{\tau} \mathrm{d} \tau^{\prime} Q\left(\hat{k}, \tau^{\prime}\right) \exp \left[\hat{k}^{4 / 3} \tau^{\prime} / S_{c}\right] .
$$

Note that we will use $R(\hat{k}, \tau)$ for non-zero $\hat{k}$ in $\S 4.4$. 
Substituting (4.23), (4.24) and (4.25), into (4.19), the powers of $k$ factor out, and we find after some changes of integration variables that

$$
\gamma=-\frac{1}{a}(1+b K)
$$

where

$$
\begin{aligned}
a= & \frac{1}{4} \iint_{\triangle_{1}} \mathrm{~d} p \mathrm{~d} q \frac{\sigma(1, p, q)}{q^{2}} p q\left[\left(1-q^{2}\right)\left(1-p^{2}+q^{2}\right) q^{-13 / 3} f(p)\right. \\
& -\left(1+p^{2}-q^{2}\right) q^{2} p^{-13 / 3} f(p)-4 q^{2} p^{-11 / 3} f(p)+\left(\left(p^{2}-q^{2}\right)^{2}\right. \\
& \left.-\left(p^{2}-3 q^{2}\right)\right) p^{-11 / 3} q^{-13 / 3} f(p)+\left(2 p^{2}\left(q^{2}+1-p^{2}\right)-4 q^{2}\right) p^{-13 / 3} f\left(\frac{q}{p}\right) \\
& \left.+\left(\left(p^{2}-q^{2}\right)^{2}-\left(p^{2}-3 q^{2}\right)\right) p^{-13 / 3} q^{-13 / 3} f\left(\frac{q}{p}\right)\right], \\
b= & \frac{1}{8} \iint_{\triangle_{1}} \mathrm{~d} p \mathrm{~d} q \frac{\sigma(1, p, q)}{q^{2}} p q\left[\left(1-q^{2}\right)\left(1-p^{2}+q^{2}\right) q^{-13 / 3} g(p, q)\right. \\
& +\left(\left(p^{2}-q^{2}\right)^{2}-\left(p^{2}-3 q^{2}\right)\right) p^{-11 / 3} q^{-13 / 3} g(p, q) \\
& \left.+\left(2 p^{2}\left(q^{2}+1-p^{2}\right)-4 q^{2}\right) p^{-11 / 3} h(p, q)\right],
\end{aligned}
$$

and

$$
\begin{aligned}
f(p) & =\int_{0}^{\infty} \mathrm{d} \tau Q(0, \tau) Q\left(0, p^{2 / 3} \tau\right), \\
g(p, q) & =\int_{0}^{\infty} \mathrm{d} \tau Q(0, \tau) Q\left(0, p^{2 / 3} \tau\right) R\left(0, q^{2 / 3} \tau\right), \\
h(p, q) & =\int_{0}^{\infty} \mathrm{d} \tau Q\left(0, p^{2 / 3} \tau\right) Q\left(0, q^{2 / 3} \tau\right) R(0, \tau) .
\end{aligned}
$$

Several of the terms in (4.28) and (4.29) contain non-integrable singularities, but these can be shown to cancel each other to give finite values for $a$ and $b$. It is such cancellations of singularities that allow Lagrangian reformulations of the DIA to give an energy spectrum with a $k^{-5 / 3}$ wavenumber dependence in the inertial range; see McComb (1990) and Leslie (1973). To evaluate the constant $\gamma$ we must specify $Q(0, \tau)$. Comparing with KG we find that their function $\check{Q}^{\dagger}(\tau)$ is given by $\check{Q}^{\dagger}(\tau)=Q\left(0, K^{-1 / 2} \tau\right)$, and we repeated their numerical calculation to determine $\mathscr{Q}^{\dagger}(\tau)$. Performing the integrals in (4.28) and (4.29), we found $a=-0.44$ and $b=0.070$, implying $\gamma=2.6$. The numerical integrations in this paper were carried out using adaptive Gauss-Konrod integration routines from the GNU Scientific Library (Galassi et al. 2001).

The shell-summed cospectrum can be evaluated using (3.11) and (4.17) to give

$$
C(k)=-\frac{4}{3} \mu \gamma \epsilon^{1 / 3} k^{-7 / 3} .
$$

Thus, the SDIP agrees with the Lumley (1967) form for the cospectrum in the inertial-convective range (1.2), and also gives the constant of proportionality. 


\subsection{The SDIP equations at low $S_{c}$}

Here we will derive the asymptotic form of the SDIP equations for $S_{c} \ll 1$. We will then compare it with the non-SDIP asymptotic result of $\S 3$. It will be useful, both here and when obtaining numerical solutions, to write (4.18) and (4.19) in non-dimensional form. We introduce the non-dimensional function $W(\hat{k})$ defined by

$$
W^{\dagger}(k, 0)=-\frac{1}{2 \pi} \gamma \epsilon^{1 / 3} k^{-13 / 3} W(\hat{k}) .
$$

The limit $\hat{k} \rightarrow 0$ represents the inertial-convective range, and so from (4.24) we find that $W(0)=1$. Some changes of integration variables result in the following linear integral equation for $W(\hat{k})$,

$$
N_{1}(\hat{k}) W(\hat{k})+N_{2}(\hat{k})+\int_{0}^{\infty} \mathrm{d} q N_{3}(\hat{k}, q) W(\hat{k} q)=0,
$$

where

$$
\begin{aligned}
N_{1}(\hat{k})= & 2 \hat{k}^{4 / 3}\left(1+\frac{1}{S_{c}}\right) \gamma \\
& -K \gamma \int_{0}^{\infty} \mathrm{d} q \int_{|1-q|}^{1+q} \mathrm{~d} p \int_{0}^{\infty} \mathrm{d} \tau \frac{p}{q} \sigma(1, p, q) p^{-11 / 3} Q\left(\hat{k} p, \tau p^{2 / 3}\right) \\
& \times\left[-\exp \left[-\hat{k}^{4 / 3} q^{2} \tau / S_{c}\right] q^{2} Q(\hat{k}, \tau) Q(\hat{k}, 0)^{-1}\right. \\
& \left.+\exp \left[-\hat{k}^{4 / 3} \tau / S_{c}\right]\left(\frac{1}{2} p^{2}\left(q^{2}+1-p^{2}\right)-q^{2}\right) Q\left(\hat{k} q, \tau q^{2 / 3}\right) Q(\hat{k} q, 0)^{-1}\right], \quad \text { (4.36) } \\
N_{2}(\hat{k})= & -K Q(\hat{k}, 0)-\frac{1}{8} K^{2} \int_{0}^{\infty} \mathrm{d} q \int_{|1-q|}^{1+q} \mathrm{~d} p \int_{0}^{\infty} \mathrm{d} \tau \frac{p}{q} \sigma(1, p, q) Q\left(\hat{k} p, \tau p^{2 / 3}\right) \\
& \times\left[\left(1-q^{2}\right)\left(1-p^{2}+q^{2}\right) Q(\hat{k}, \tau) Q(\hat{k} p, 0)^{-1} R\left(\hat{k} q, \tau q^{2 / 3}\right) q^{-13 / 3}\right. \\
& +\left(\left(p^{2}-q^{2}\right)^{2}-p^{2}+3 q^{2}\right) Q(\hat{k}, \tau) Q(\hat{k}, 0)^{-1} R\left(\hat{k} q, \tau q^{2 / 3}\right) p^{-11 / 3} q^{-13 / 3} \\
& \left.+\left(2 p^{2}\left(q^{2}+1-p^{2}\right)-4 q^{2}\right) Q\left(\hat{k} q, \tau q^{2 / 3}\right) Q(\hat{k} q, 0)^{-1} R(\hat{k}, \tau) p^{-11 / 3}\right], \quad(4.37) \\
N_{3}(\hat{k}, q)= & -\frac{1}{4} \gamma K q^{-13 / 3} \int_{|1-q|}^{1+q} \mathrm{~d} p \int_{0}^{\infty} \mathrm{d} \tau \frac{p}{q} \sigma(1, p, q) \\
& \times\left[\left(1-q^{2}\right)\left(1-p^{2}+q^{2}\right) Q(\hat{k}, \tau) Q\left(\hat{k} p, \tau p^{2 / 3}\right) Q(\hat{k} p, 0)^{-1} \exp \left[-\hat{k}^{4 / 3} q^{2} \tau / S_{c}\right]\right. \\
& +\left(\left(p^{2}-q^{2}\right)^{2}-p^{2}+3 q^{2}\right) p^{-11 / 3} Q\left(\hat{k} p, \tau p^{2 / 3}\right) \\
& \times\left(Q(\hat{k}, \tau) Q(\hat{k}, 0)^{-1} \exp \left[-\hat{k}^{4 / 3} q^{2} \tau / S_{c}\right]\right. \\
& \left.+Q\left(\hat{k} q, \tau q^{2 / 3}\right) Q(\hat{k} q, 0)^{-1} \exp \left[-\hat{k}^{4 / 3} \tau / S_{c}\right]\right) \\
& \left.-p^{2}\left(1+q^{2}-p^{2}\right) Q(\hat{k}, \tau) Q\left(\hat{k} q, \tau q^{2 / 3}\right) Q(\hat{k} q, 0)^{-1} \exp \left[-\hat{k}^{4 / 3} p^{2} \tau / S_{c}\right]\right] . \quad \text { (4.38) }
\end{aligned}
$$

Note that $R(\hat{k}, \tau)$ is defined by (4.26), and that we have effectively combined (4.18) and (4.19) into one equation for $W(\hat{k})$.

For clarity of analysis when taking the small $S c$ limit we follow Goto \& Kida (1999) by introducing the rescaled quantities $\hat{k}=\hat{k}_{s} S_{c}^{\alpha}$ and $W(\hat{k})=W_{s}\left(\hat{k}_{s}\right) S_{c}^{\beta}$, where $\hat{k}_{s}$ and $W_{s}$ are assumed to be order unity as $S_{c} \rightarrow 0$. The Kolmogorov wavenumber and the Obukhov-Corrsin wavenumber then correspond to $\alpha=0$ and $\alpha=3 / 4$, respectively. We wish to consider wavenumbers $k \gg k_{C}$ and so $\alpha<3 / 4$. Typical exponential factors involving $S_{c}$ are rewritten as

$$
\exp \left[-\hat{k}^{4 / 3} \tau / S_{c}\right]=\exp \left[-\hat{k}_{s}^{4 / 3} \tau S_{c}^{4 \alpha / 3-1}\right] \simeq 0,
$$


because $4 \alpha / 3-1<0$. Therefore in (4.35) we can neglect $N_{3}$, and $N_{1}$ is approximated by

$$
N_{1} \simeq 2 \hat{k}_{s}^{4 / 3} S_{c}^{4 \alpha / 3-1} \gamma .
$$

Care must be taken with the function $R(\hat{k}, \tau)$, which is written using the rescaled quantities as

$$
R(\hat{k}, \tau)=\int_{0}^{\tau} \mathrm{d} \tau^{\prime} Q\left(\hat{k}, \tau^{\prime}\right) \exp \left[-\hat{k}_{s}^{4 / 3} S_{c}^{4 \alpha / 3-1}\left(\tau-\tau^{\prime}\right)\right] .
$$

Noting that $\tau \geqslant \tau^{\prime}$ we have

$$
N_{2} \simeq-K Q\left(\hat{k}_{s} S_{c}^{\alpha}, 0\right) .
$$

The relevant scaling for $W_{s}\left(\hat{k}_{s}\right)$ is then $\beta=-4 \alpha / 3+1$, with the result

$$
W_{s}\left(\hat{k}_{s}\right)=\frac{K Q\left(\hat{k}_{s} S_{c}^{\alpha}, 0\right)}{2 \hat{k}_{s}^{4 / 3} \gamma} .
$$

Returning to unscaled variables, we have

$$
W(\hat{k})=\frac{K Q(\hat{k}, 0) S_{c}}{2 \hat{k}^{4 / 3} \gamma}, \quad k \gg k_{C} .
$$

The corresponding form for the shell-summed cospectrum is

$$
C(k)=-\frac{2 \mu}{3 \kappa k^{2}} E(k), \quad k \gg k_{C},
$$

and for $k_{C} \ll k \ll k_{K}$ we recover (3.24). Therefore the SDIP equation is consistent with the asymptotic result of $\S 3.3$ for the inertial-diffusive range that was derived using the simpler Batchelor et al. (1959) type analysis. The SDIP asymptotic form (4.45) is more general since it applies in the viscous-diffusive range also. The SDIP equations are solved numerically in $\S 4.5$ and the asymptotic form (4.45) is verified.

\subsection{Numerical solution}

Here we will solve (4.35) numerically for a range of values of $S c$. Equation (4.35) is an inhomogeneous Fredholm integral equation of the second kind. As written, the integrals giving $N_{1}(\hat{k}) W(\hat{k})$ and $\int_{0}^{\infty} \mathrm{d} q N_{3}(\hat{k}, q) W(\hat{k} q)$ do not converge, because of a non-integrable singularity as $(p \rightarrow 0, q \rightarrow 1)$. These singularities do cancel each other, but involve the unknown function $W(\hat{k})$ in a way that makes adding and subtracting the singularity difficult. Therefore, the method of solution chosen was to use a Newton-Raphson solver. This simplifies the problem because calculation of the residuals requires finding the left-hand side of the equation, but not each individual term. Although the equation is linear, in practice two iterations were required for good accuracy.

The $q$ integration was performed using a Gauss-Jacobi rule, and the $p$ and $\tau$ integrations were performed using adaptive Gauss-Konrod integration. The Jacobian required for the Newton-Raphson solver was calculated using finite differences, and interpolations were performed using cubic and bicubic spline interpolation for $W(\hat{k})$ and $Q(\hat{k}, \tau)$, respectively.

The results were found to be converged when the integral equation was evaluated at twenty points, and when for the $q$ integration the integrand was evaluated at twenty-four points. The integral equation was evaluated at the same points as where $W(\hat{k})$ was stored, but these points were chosen to be different from the quadrature 

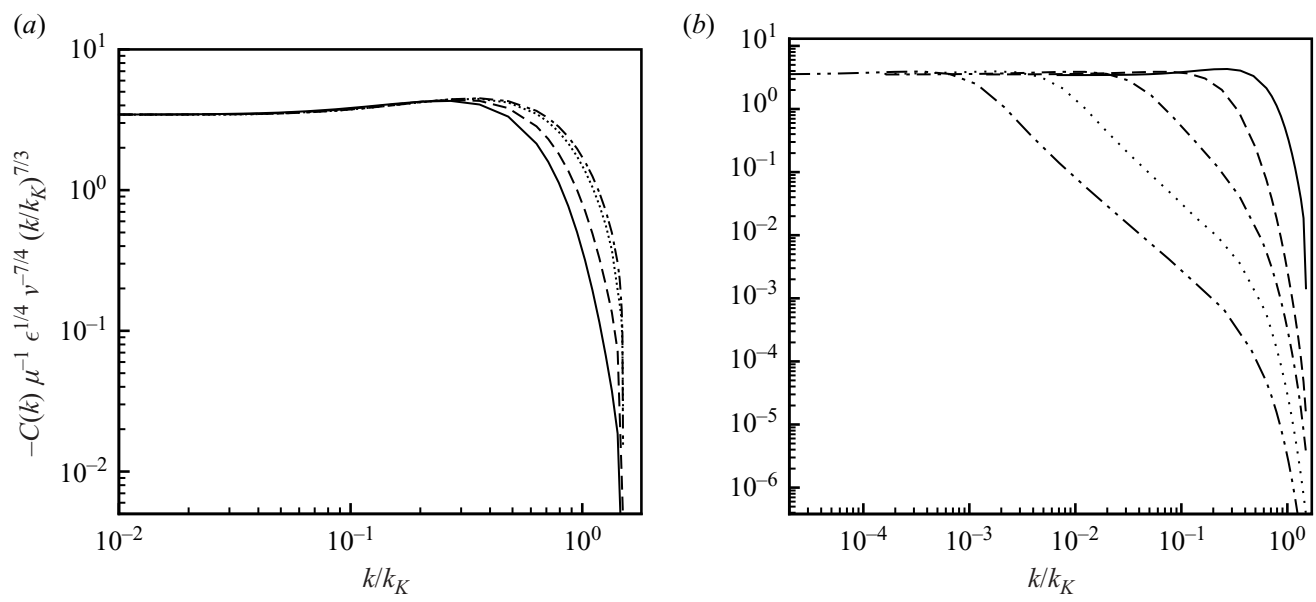

FIGURE 1. SDIP results for the compensated shell-summed velocity-scalar cospectrum: (a) $S_{c}=1$ (solid), 2 (dashed), 10 (dotted), and 100 (dash-dotted). (b) $S_{c}=1$ (solid), $10^{-1}$ (dashed), $10^{-2}$ (dash-dotted), $10^{-3}$ (dotted), and $10^{-4}$ (dash-dot-dotted).

points for the $q$ integration. This was necessary because $W$ enters the $q$ integral as $W(\hat{k} q)$, and so, for small $\hat{k}$, the $q$ integration must extend to large values for accuracy. A rescaling to a form with $W(q)$ was not possible without the undesirable effect of making the location of the singular points a function of $\hat{k}$.

Solving (4.35) obviously requires knowledge of the two-time two-point velocity statistics through the function $Q(\hat{k}, \tau)$. This was obtained by repeating the SDIP calculation of $\mathrm{KG}$, involving the solution of a coupled system of a nonlinear integral equation, and a second-order integro-differential equation. Our results for $Q(\hat{k}, 0)$ were found to match their reported results to within graphing accuracy.

The shell-summed cospectrum is related to $W(\hat{k})$ by

$$
C(k)=-\frac{4}{3} \mu \gamma \epsilon^{1 / 3} k^{-7 / 3} W(\hat{k}),
$$

and figure $1(a)$ shows the results for the compensated shell-summed cospectrum for a range of $S_{c}>1$. As might be expected, the cospectrum decays more slowly for increasing $S_{c}$, but nonetheless smoothly approaches the inertial-convective limit in each case. Note that we did not enforce the inertial-convective limit, $W(0)=1$, so that this condition is a check on the consistency of our results with the inertial-convective calculation. There is a characteristic bump structure, which is located at approximately $0.3 k_{K}$ for $S_{c}=1$. The cospectrum quickly reaches an asymptotic form for large $S_{c}$, and when graphed there was no visible difference between the cospectra at $S_{c}=100$ and 1000. As was discussed in $\S 3$, there seems to be no power-law behaviour in the viscous-convective range. The compensated shell-summed cospectrum is shown in figure $1(b)$ for a range of small $S_{c}$. Again there is a smooth approach to the inertial-convective limit in each case.

In figure 2 we compare the numerical solution to the SDIP equation at a range of $S_{c} \leqslant 1$ with the inertial-diffusive power law form (3.24). In contrast to figure $1(b)$, we have scaled the wavenumber with the Obukhov-Corrsin wavenumber rather than the Kolmogorov wavenumber, so that the normalized inertial-diffusive asymptotic result is independent of $S_{c}$. The approach to the power law form is evident for the lower $S_{c}$ cospectra. In figure 3 we make a comparison at $S_{c}=10^{-4}$ of the SDIP asymptotic 


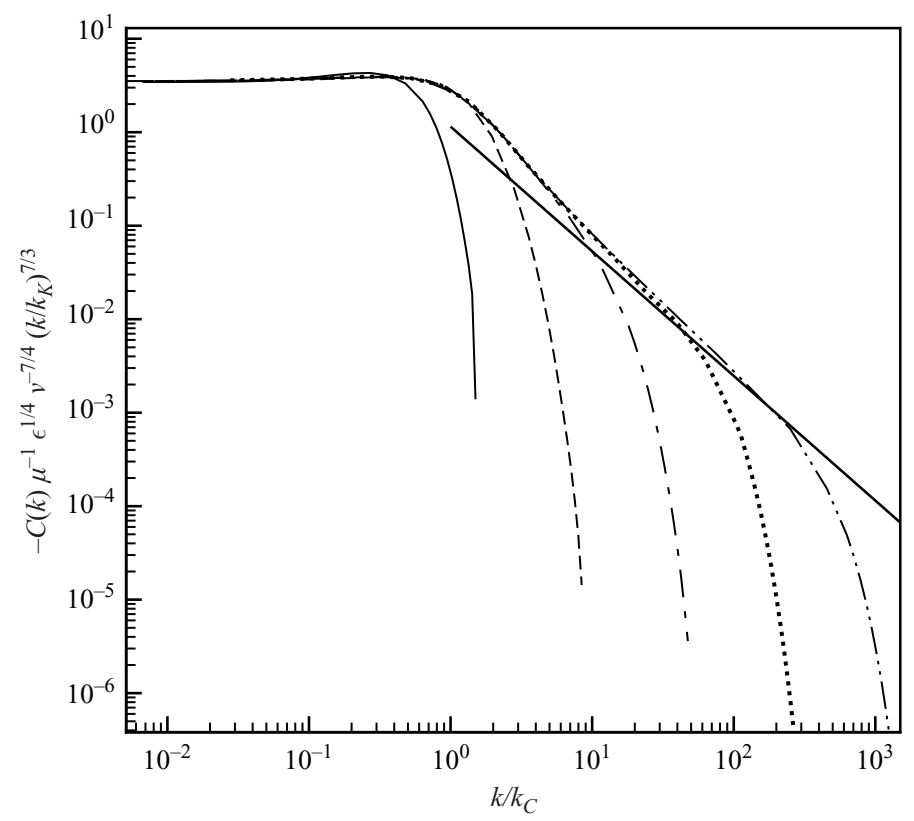

FIGURE 2. SDIP results for the compensated shell-summed velocity-scalar cospectrum at low $S_{c}$ (same key as figure $1 b$ ). Here the wavenumber has been normalized with the Obukhov-Corrsin wavenumber, and the thick solid line shows the inertial-diffusive asymptotic result given by (3.24).

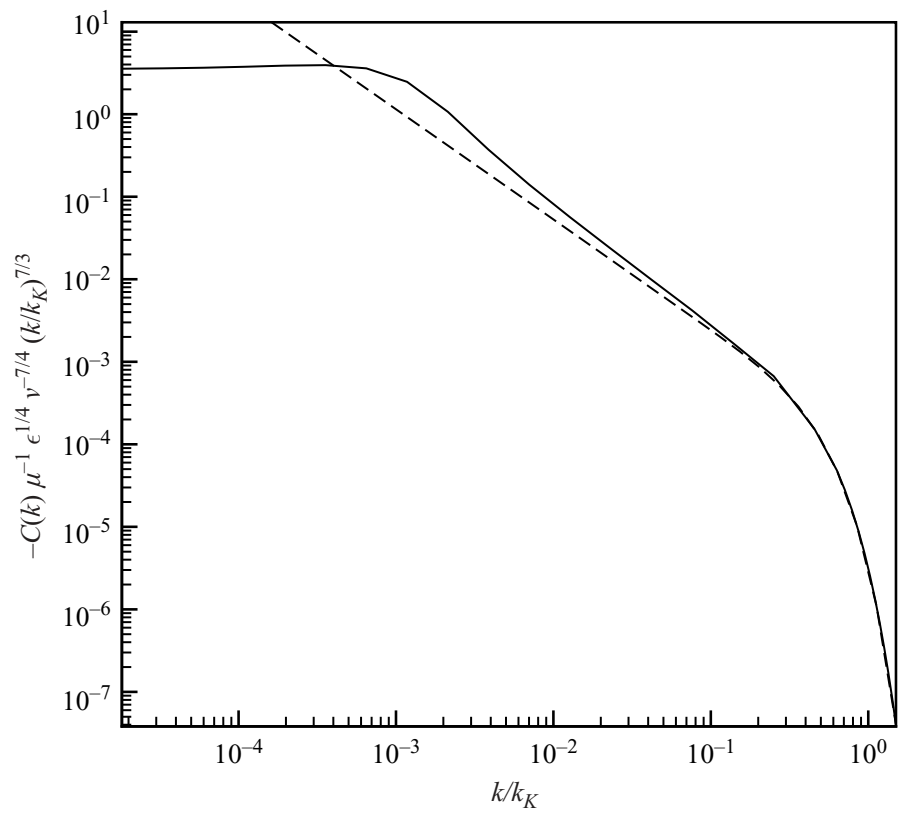

FIGURE 3. SDIP result for the compensated shell-summed velocity-scalar cospectrum at $S_{c}=10^{-4}$ (solid) compared with the SDIP asymptotic form given by (4.45) (dashed). 


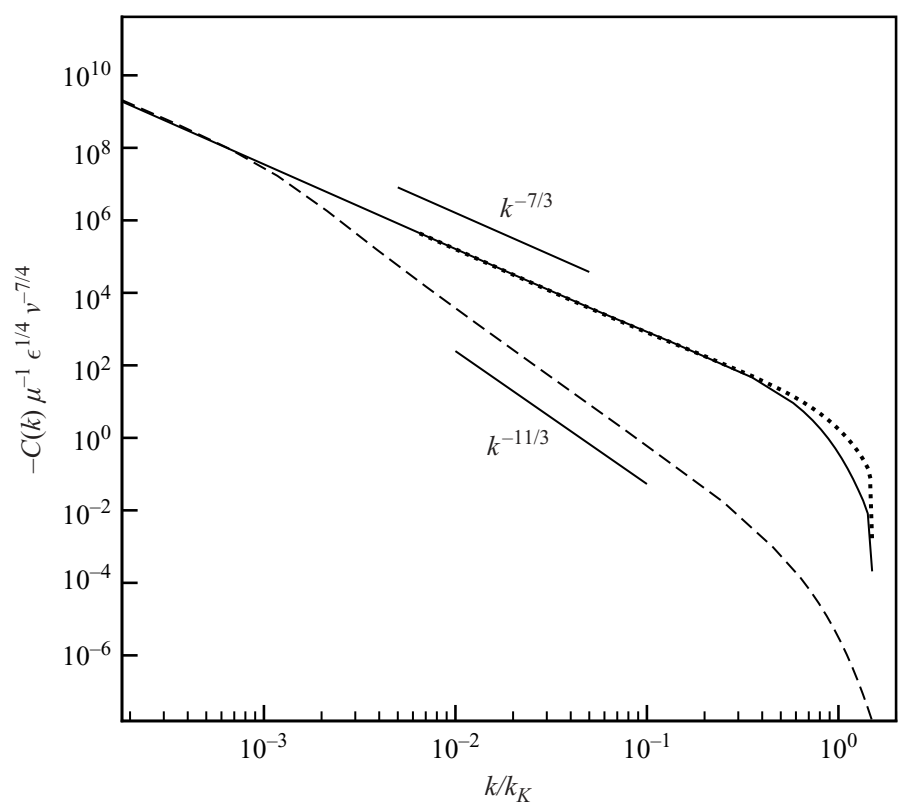

FIGURE 4. SDIP results for the shell-summed velocity-scalar cospectrum (uncompensated) at $S_{c}=10^{-4}$ (dashed), 1 (solid), and $10^{4}$ (dotted). The power laws $k^{-7 / 3}$ and $k^{-11 / 3}$ are also shown for reference.

form (4.45), and the SDIP numerical result. The agreement of the numerical result with the SDIP asymptotic form is excellent in the viscous-diffusive range.

Finally, to summarize our results for the effect of $S_{c}$ on the cospectrum, figure 4 shows the shell-summed cospectrum in uncompensated form for $S_{c}=10^{-4}, 1,10^{4}$, making clear the two distinct power law ranges for small $S_{c}$. At large $S_{c}$, the relatively small effect of changes in $S_{c}$ is evident, at least when the wavenumber is scaled with the Kolmogorov wavenumber, corresponding to varying the diffusivity with the viscosity and energy dissipation held constant. Comparison with experiment, DNS, and the stretched-spiral vortex model at $S_{c}$ of order unity will be made in $\S 5.2$.

\section{Numerical experiments}

Here, results are reported for a DNS at $S c$ order unity and an LES at low $S c$. In both the DNS and the LES, a statistically homogeneous and isotropic velocity field mixed a passive scalar with a mean scalar gradient.

\subsection{Direct numerical simulation}

A DNS was performed on the QSC supercomputer at a Taylor Reynolds number, $R_{\lambda}$, of 265 and $S c$ of 0.7 , and the velocity-scalar cospectrum was calculated. The velocity field was evolved according to the Navier-Stokes equations, and the scalar was evolved using the advection-diffusion equation. Statistics were calculated at a statistically stationary state achieved by forcing the velocity field at low wavenumbers. A pseudospectral method was used, and further details of the numerical method may be found in O'Gorman \& Pullin (2004). Results from an additional simulation at $R_{\lambda}=167$ are also reported, and parameters describing the simulations are summarized in table 1 . The Courant number used was relatively low because the time step was fixed so that two-time statistics could be collected easily. The value of $k_{\max } / k_{K}$ used 


$\begin{array}{ccccccccc}\text { Grid } & R_{\lambda} & T_{\text {stat }} / T_{\text {eddy }} & S_{c} & k_{\max } / k_{K} & R_{l} & \overline{\theta^{2}} /\left(\mu l_{\epsilon}\right)^{2} & k_{0} l & C \\ 512^{3} & 265 & 10.5 & 0.7 & 1.05 & 1901 & 0.45 & 1.00 & 0.48 \\ 256^{3} & 167 & 9.3 & 0.7 & 1.00 & 779 & 0.38 & 0.99 & 0.51\end{array}$

TABLE 1. Simulation parameters for the stationary period of the DNS. $T_{\text {eddy }}$ is the eddy turnover time, $T_{\text {stat }}$ is the time over which the statistics are collected, $k_{\max }$ is the largest dynamically important wavenumber, $R_{l}$ is the Reynolds number based on the integral length scale $l$, the turbulent length scale is $l_{\epsilon}=u_{r m s}^{3} / \epsilon$ where $u_{r m s}$ is the root-mean-square velocity, $k_{0}$ is the smallest wavenumber, and $C$ is the Courant number.
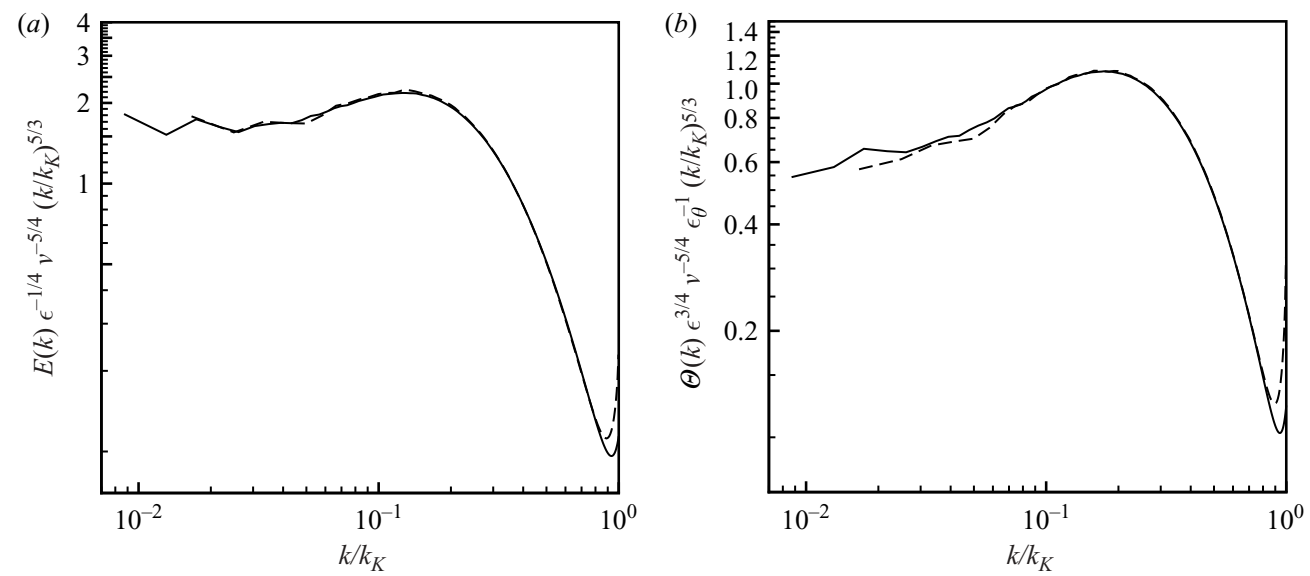

Figure 5. Compensated spectra from the DNS at $512^{3}$ (solid line) and $256^{3}$ (dashed line): (a) energy spectra, (b) scalar spectra.

is at the low end of the range of commonly used values when passive scalar statistics are collected at $S c=0.7$.

For both simulations, the shell-summed energy spectra are shown in figure 5( $a$ ), and the shell-summed scalar spectra are shown in figure $5(b)$. These spectra are shown in compensated form, and are useful as a reference when considering the velocity-scalar cospectrum. A small inertial range and a bump in the dissipation range are apparent for the energy spectra. The slope of the scalar spectra in the convective range is shallower than $k^{-5 / 3}$, although it could be argued that a true inertial-convective range is not apparent at these Reynolds numbers.

The shell-summed velocity-scalar cospectra for the two simulations are shown in compensated form in figure $6(a)$. The cospectral slope is shallower than $k^{-7 / 3}$ in the inertial-convective range. Note that the cospectra for the simulations at $R_{\lambda}$ of 265 and 167 do not exactly coincide for intermediate or large wavenumber, at least with the normalization used. This is in contrast to the energy and scalar spectra in figures $5(a)$ and $5(b)$ which appear to show a universal ( $R_{\lambda}$ independent) wavenumber range. Mydlarski (2003) also found a dependence on $R_{\lambda}$ for the related one-dimensional heat flux structure function in the inertial-convective range. Further investigation at a range of different $R_{\lambda}$ seems warranted.

The one-dimensional cross-spectrum inequality is closely related to the spectral coherence, see Bendat \& Piersol (1986). Similarly, using the the cospectral inequality 

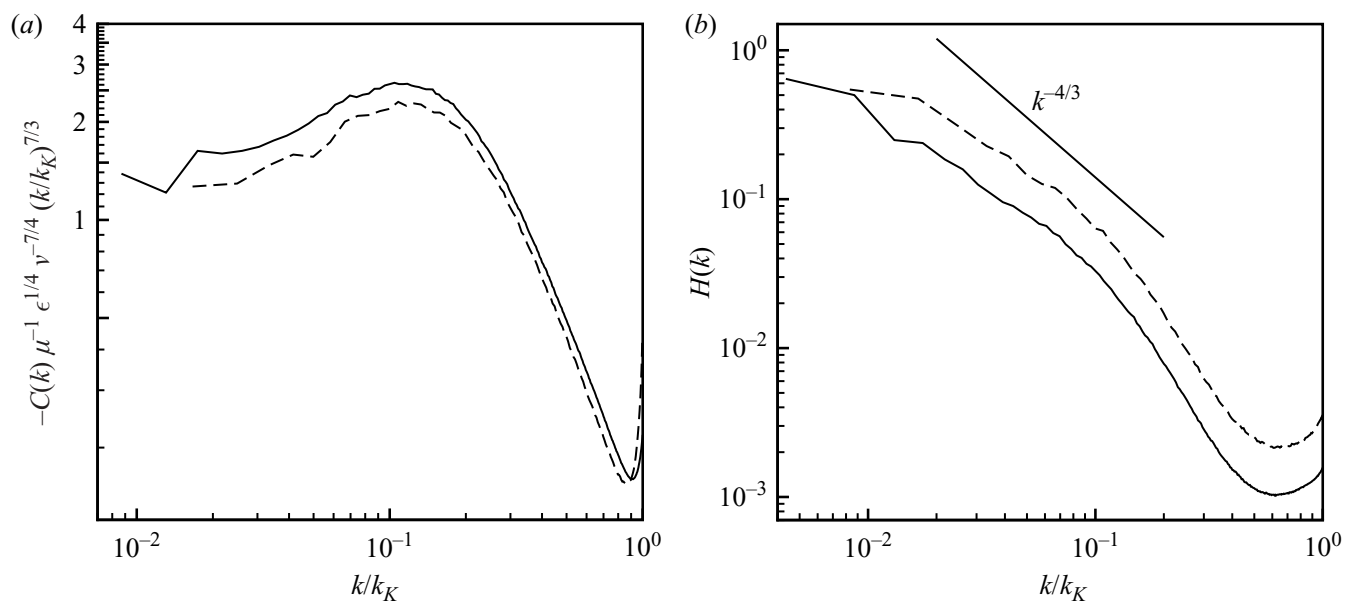

FIGURE 6. DNS results at $512^{3}$ (solid line) and $256^{3}$ (dashed line): (a) compensated shell-summed velocity-scalar cospectra, $(b)$ shell-summed spectral coherence compared with a $k^{-4 / 3}$ power law.

(3.14) as a guide, we can define a shell-summed spectral coherence,

$$
H(k)=\frac{3 C(k)^{2}}{2 E(k) \Theta(k)},
$$

so that $H(k) \leqslant 1$. We note that whereas the definition of the one-dimensional spectral coherence involves the quadrature spectrum, the shell-summed cross-spectrum is real by definition. The magnitude of $H(k)$ is a measure of how correlated the scalar and velocity Fourier components are at a given wavenumber, and is clearly a measure of the approach to isotropy at small scales. In figure $6(b)$, we plot $H(k)$ from the DNS, noting that if the Lumley scaling form (1.2) holds, then $H(k)$ will have a $k^{-4 / 3}$ wavenumber-dependence in the inertial-convective range. The slope in the inertialconvective range is shallower than $k^{-4 / 3}$. The maximum value of $H(k)$ is approximately 0.6 and this occurs at the lowest wavenumber. Note that we have omitted the lowest wavenumber for each simulation in figures $5(a), 5(b)$ and $6(a)$ for readability.

Unlike for the normalized cospectra in figure $6(a)$, we do not expect the spectral coherence functions to coincide at different $R_{\lambda}$. Assume for the moment that the normalization used in figure $6(a)$, namely $C(k) \mu^{-1} \epsilon^{1 / 4} v^{-7 / 4}$, gives an approximately universal form for the cospectrum when plotted against $k / k_{K}$. We can also use the standard universal forms for the energy and scalar spectra as these give good collapse of the energy and scalar spectra in figures $5(a)$ and $5(b)$. Then the shell-summed spectral coherence should vary in the same way with $R_{\lambda}$ as the non-dimensional number $\epsilon_{\theta}^{-1} \mu^{2} v$, and this can be expected to have a strong $R_{\lambda}$ dependence.

\subsection{Comparison of DNS results with theory and experiment}

We make a comparison of the DNS results for the cospectrum with the SDIP result of $\S 4$, the stretched-spiral vortex model of O'Gorman \& Pullin (2003), and the experimental result of Mydlarski \& Warhaft (1998). We take $S_{c}=0.7$, except in the case of the stretched-spiral vortex model where $S_{c}$ is restricted to be unity. For this model we also consider only the component of the cospectrum owing to axial motion in the vortex structures, see O'Gorman \& Pullin (2003) for further details. The experimental result was for the one-dimensional cospectrum of the velocity $u_{1}$ 


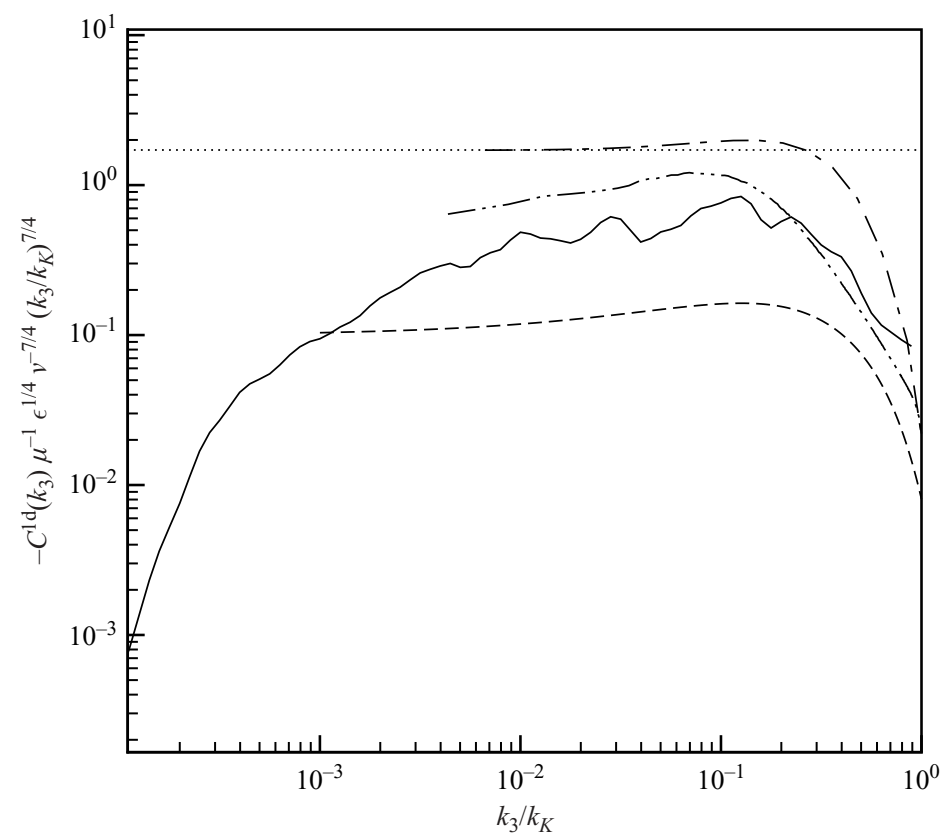

FIGURE 7. Comparison of one-dimensional velocity-scalar cospectra from the stretched-spiral vortex model (dashed), experimental data from Mydlarski \& Warhaft (1998) at $R_{\lambda}=582$ (solid), DNS at $R_{\lambda}=265$ (dash-dot-dotted), SDIP inertial-convective (dotted), and SDIP (dash-dotted).

and temperature, $C^{1 d}\left(k_{3}\right)$, at $R_{\lambda}$ of 582 . Note that in the experiment, the direction of the scalar gradient, and hence the scalar flux, was perpendicular to the direction in which the cospectrum was measured. The experimental data were rather noisy, and so we have applied a one-third octave smoothing filter. To make the comparison, we convert the SDIP and stretched-spiral vortex model shell-summed cospectra to one-dimensional cospectra using the following formula derived in O'Gorman \& Pullin (2003),

$$
C^{1 d}\left(k_{3}\right)=\frac{3}{4} \int_{k_{3}}^{\infty} \frac{k^{2}+k_{3}^{2}}{k^{3}} C(k) \mathrm{d} k .
$$

The one-dimensional cospectrum was calculated directly in the DNS.

The cospectra are shown in figure 7 in compensated form, where we have also shown a straight line representing the inertial-convective SDIP result. The DNS cospectrum was calculated by time-averaging a sequence of normalized spectra, and so a rough estimate of the error is given by the time variation at a given wavenumber. At $k / k_{k}=0.05$ we found that the standard deviation of the compensated cospectrum $C^{1 d}\left(k_{3}\right) \mu^{-1} \epsilon^{1 / 4} v^{-7 / 4}\left(k_{3} / k_{K}\right)^{7 / 3}$ was 0.14 . It is difficult to estimate the error in the experimental cospectrum, but we note that the unfiltered compensated cospectrum had excursions of the order of half a decade in the inertial-convective range.

The shapes of the cospectra are similar in all cases, although the SDIP cospectrum is closer than the other cospectra to a $k^{-7 / 3}$ power law in the inertial-convective range. The DNS has a similar spectral slope to that of the experimental result which was reported as $k^{-2}$. The SDIP cospectrum seems to be too large in the inertialconvective range, and in this context it is worth noting that the SDIP value for the Obukhov-Corrsin differs from experimental values by a factor of about a half. The 
discrepancy may be related to the strong assumption used in the SDIP closure of statistical independence between the position function and several other variables.

\subsection{Large-eddy simulation at low $S_{c}$}

A large-eddy simulation (LES) was performed to verify the asymptotic results (3.17) and (3.24) for the scalar spectrum and velocity-scalar cospectrum, respectively, at low $S c$ in the inertial-diffusive range. Our simulation is similar to the LES of Chasnov (1991), where a comparison was made with the asymptotic form (3.17) for the scalar spectrum. Chasnov found excellent agreement in the far inertial-diffusive range, but the agreement in the near inertial-diffusive range was not as good. As was the case for Chasnov, we do not need a subgrid model for the scalar because we resolve the diffusive range. Our subgrid model for the velocity is different from that used by Chasnov.

A statistically stationary state was reached in the same way as in the DNS by forcing the velocity at the low wavenumbers, and with a mean scalar gradient acting as the source for the scalar variance. The resolution used was $64^{3}$ gridpoints, at an $R_{\lambda}$ of 1500 , and with $S c=2 \times 10^{-4}$. Statistics were collected over thirty large-eddy turnover times, and time-averaged spectra are reported. The details of the velocity forcing and the pseudospectral method are the same as in Pullin (2000). We used the stretchedvortex SGS model of Misra \& Pullin (1997), with vortex alignment according to the locally resolved strain rates (model 1a), and a spiral-vortex-type energy spectrum at the subgrid scales. Evaluation of the second-order velocity structure function was used to calculate the subgrid energy, see Voelkl, Pullin \& Chan (2000) and Pullin (2000) for further details. This LES method has the advantage of dynamically giving a value for the Kolmogorov constant. The time-averaged value was $K=1.40$ with a standard deviation of 0.05 , which may be compared with the more commonly accepted value of $K=1.6$ (Sreenivasan 1995) We evaluate expressions (3.17) and (3.24) using the value for $K$ dynamically calculated by the LES because we need a value of $K$ that is consistent with the energy spectrum in the LES in order to test the validity of these asymptotic expressions fairly.

The LES result for the velocity-scalar cospectrum, and the asymptotic result given by (3.24) are compared in figure 8. The agreement is reasonable for wavenumbers in the inertial-diffusive range, although the asymptotic result is lower than the LES result. The LES result for the scalar spectrum, and the asymptotic result given by (3.17) are compared in figure $9(a)$. The Obukhov-Corrsin wavenumber corresponds to $k_{C} / k_{K} \simeq 0.002$, and so we can see that in the inertial-diffusive range the agreement is quite good. Thus, LES seems to confirm the asymptotic results for both the velocityscalar cospectrum and the scalar spectrum, although of course any mechanism that requires a resolved viscous range has been neglected in our LES. For reference, we show in figure $9(b)$ the energy spectrum from the LES compared with the inertial-range form (3.23), where we have again used the Kolmogorov constant given by the LES.

\section{Concluding remarks}

Several properties of the velocity-scalar cospectrum were examined in this study. In the inertial-convective range, the SDIP result for the cospectrum was shown to agree with the Lumley (1967) scaling. A comparison was made of the experimental data of Mydlarski \& Warhaft (1998) with the result from the SDIP, the stretched-spiral vortex model result of O'Gorman \& Pullin (2003), and the DNS result. The cospectral slopes in the inertial-convective range were all found to be shallower than $k^{-7 / 3}$, where 


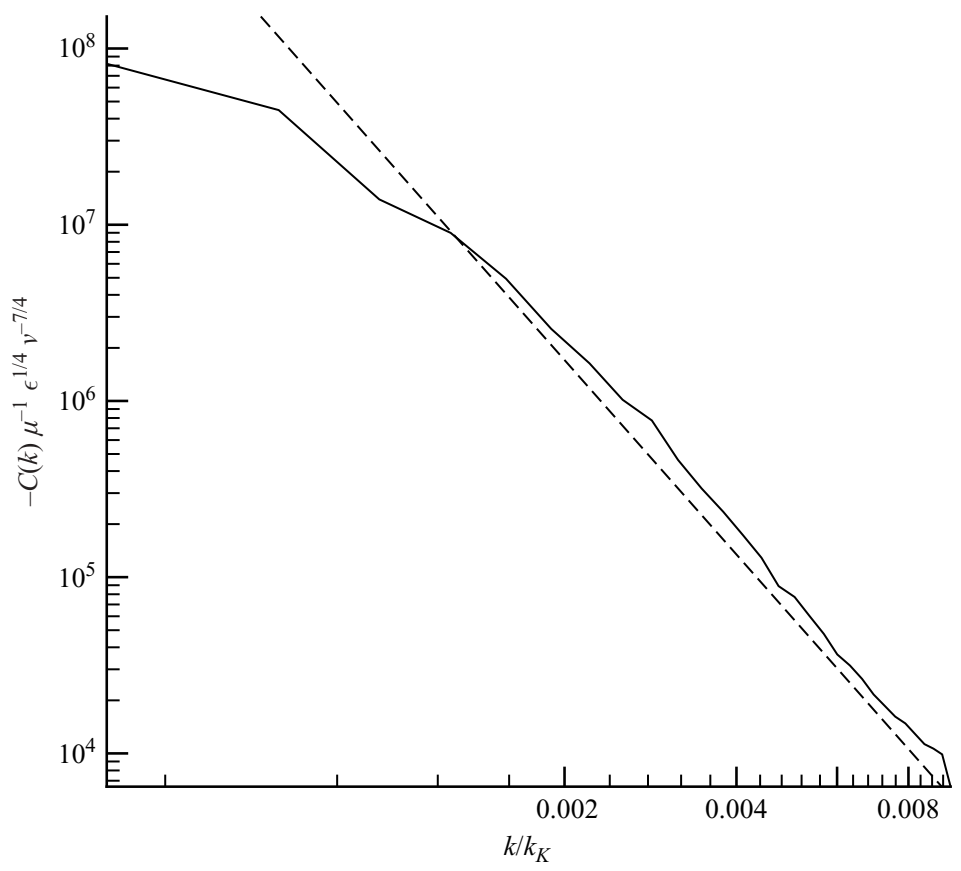

FIGURE 8. Comparison of the velocity-scalar cospectrum from the LES (solid) and the low $S_{c}$ asymptotic form given by (3.24) (dashed).
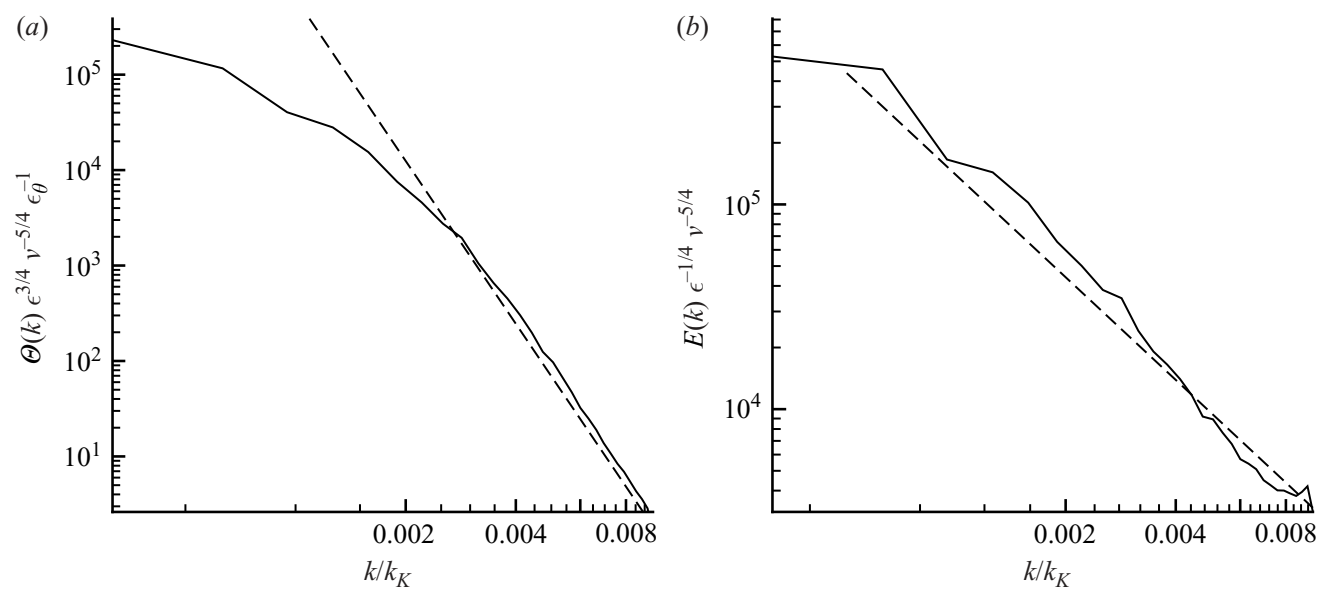

Figure 9. (a) Comparison of the scalar spectrum from the LES (solid) and the low $S_{c}$ asymptotic form given by (3.17) (dashed). (b) Comparison of the energy spectrum from the LES (solid) and the inertial range form given by (3.23) (dashed).

Mydlarski \& Warhaft had found $k^{-2}$ at their largest $R_{\lambda}$. The decay of the cospectrum with wavenumber in the inertial-convective range was found to be faster than that of the scalar or the energy spectra, and this is consistent with the idea of an approach to isotropy at small scales.

The effect on the cospectrum of different $S c$ was investigated. An upper bound was derived for the magnitude of the cospectrum in terms of the shell-summed energy and scalar spectra, with the implication that at high $S c$ the cospectrum would 
decay exponentially with wavenumber in the viscous-convective range. This limits the possible contribution of sub-Kolmogorov length scales to the mean scalar flux, a result that may be important in subgrid modelling of the scalar flux. At low $S c$, a new asymptotic form was found for the velocity-scalar cospectrum, with a $k^{-11 / 3}$ power law wavenumber-dependence in the inertial-diffusive range. The inertial-diffusive asymptotic forms for the cospectrum and the scalar spectrum were confirmed using LES, and are in principle subject to experimental verification, perhaps using a liquid metal. At least in this regime, the forcing of the scalar fluctuation by the mean scalar gradient is important at large wavenumber for both the scalar spectrum and the velocity-scalar cospectrum. Although the derivation of the SDIP equation for the cospectrum was complicated, one advantage of the method is that it was then relatively inexpensive to investigate a wide range of $S c$. Using the SDIP equation, the asymptotic form of the cospectrum in the inertial-diffusive range was confirmed and extended to the viscous-diffusive range. At high $S c$, the SDIP result for the cospectrum was indeed found to decay exponentially in the viscous-convective range, as was expected from the cospectrum inequality.

One possibility for future work, suggested by the DNS results described here, is an investigation of the Reynolds-number dependence of the cospectrum when plotted using Kolmogorov scaling. Another possibility for future work is the calculation of the scalar spectrum using the SDIP in the case of a mean scalar gradient. The SDIP equation for the passive scalar spectrum would then involve the velocity-scalar cospectrum, so that in a sense some of the work has already been done.

P.A.OG. and D.I.P. were supported in part by the National Science Foundation under Grant CTS-0227881. The DNS was performed on the QSC supercomputer, and was supported by the Academic Strategic Alliances Program of the Accelerated Strategic Computing Initiative (ASCI/ASAP) under subcontract no. B341492 of DOE contract W-7405-ENG-48.

\section{Appendix A}

Here we will show how the Batchelor et al. (1959) result for the scalar spectrum in the inertial-diffusive range is modified by the presence of a mean scalar gradient. In the inertial-diffusive range, we have shown in $\S 3.3$ that an approximate equation for $\tilde{\theta}(\boldsymbol{k}, t)$ is given by neglecting the time derivative in (3.19). Multiplying by a similar expression for $\tilde{\theta}(-\boldsymbol{k}, t)$, and taking an ensemble average gives

$$
\begin{aligned}
\overline{\tilde{\theta}(\boldsymbol{k}, t) \tilde{\theta}(-\boldsymbol{k}, t)} \kappa^{2} k^{4}= & \mu^{2} \tilde{\tilde{u}}_{1}(\boldsymbol{k}, t) \tilde{u}_{1}(-\boldsymbol{k}, t) \\
& -\left(\frac{2 \pi}{L}\right)^{6} \sum_{\boldsymbol{p}} \sum_{\boldsymbol{q}} p_{j} q_{i} \overline{\tilde{u}_{j}(-\boldsymbol{k}-\boldsymbol{p}, t) \tilde{u}_{i}(\boldsymbol{k}-\boldsymbol{q}, t) \tilde{\theta}(\boldsymbol{p}, t) \tilde{\theta}(\boldsymbol{q}, t)} \\
& +2 \mu\left(\frac{2 \pi}{L}\right)^{3} \operatorname{Re}\left(\mathrm{i} \sum_{\boldsymbol{q}} q_{i} \overline{\tilde{u}_{i}(-\boldsymbol{k}-\boldsymbol{q}, t) \tilde{\theta}(\boldsymbol{q}, t) \tilde{u}_{1}(\boldsymbol{k}, t)}\right) .
\end{aligned}
$$

Again using $|\boldsymbol{q}| \ll k,|\boldsymbol{p}| \ll k$, and assuming the statistical independence of modes at wavenumber $\boldsymbol{q}$ or $\boldsymbol{p}$ with modes at $\boldsymbol{k}-\boldsymbol{q}, \boldsymbol{k}$, or $\boldsymbol{k}-\boldsymbol{p}$, we find

$$
\begin{aligned}
\overline{\tilde{\theta}(\boldsymbol{k}, t) \tilde{\theta}(-\boldsymbol{k}, t)} \kappa^{2} k^{4}= & \mu^{2} \overline{\tilde{u}_{1}(\boldsymbol{k}, t) \tilde{u}_{1}(-\boldsymbol{k}, t)} \\
& -\left(\frac{2 \pi}{L}\right)^{6} \sum_{\boldsymbol{p}} \sum_{\boldsymbol{q}} p_{j} q_{i} \overline{\tilde{u}_{j}(-\boldsymbol{k}-\boldsymbol{p}, t) \tilde{u}_{i}(\boldsymbol{k}-\boldsymbol{q}, t)} \overline{\tilde{\theta}(\boldsymbol{p}, t) \tilde{\theta}(\boldsymbol{q}, t) .}
\end{aligned}
$$


This may be simplified to

$$
\kappa^{2} k^{4} \tilde{Z}(\boldsymbol{k}, t, t)=\mu^{2} \tilde{V}_{11}(\boldsymbol{k}, t, t)+\tilde{V}_{11}(\boldsymbol{k}, t, t)\left(\frac{2 \pi}{L}\right)^{3} \sum_{q} q_{j} q_{j} \tilde{Z}(\boldsymbol{q}, t, t) .
$$

The sum over $\boldsymbol{q}$ can be related to the scalar dissipation. Taking the limit $L \rightarrow \infty$, and performing a surface integral in wavenumber space leads to

$$
\Theta(k)=\frac{2}{3} E(k) \kappa^{-2} k^{-4}\left(\mu^{2}+\frac{\epsilon_{\theta}}{2 \kappa}\right) .
$$

We are in the inertial range, and so using the appropriate form of the energy spectrum (3.23) gives the result (3.17), which reduces to the Batchelor et al. (1959) result when the mean scalar gradient, $\mu$, is set to zero.

\section{Appendix B}

This Appendix outlines the derivation of the closed SDIP equations for the velocityscalar cospectrum. Further details of the derivation are given in Appendices C, D, and E. $\uparrow$ The exact evolution equations for one-and two-point statistics are derived in Fourier space in $\S \S$ B.1 and B.2. The direct-interaction decompositions are made in $\S$ B.3, and the closed equations are derived in $\S$ B.4. Finally, the simplification of the equations for the statistically stationary and axisymmetric case is described in $\S$ B.5.

\section{B.1. Equations in Fourier space}

We begin by writing the governing equations in Fourier space. The incompressibility of the velocity field implies that $k_{i} \tilde{u}_{i}(\boldsymbol{k}, t)=0$. The Navier-Stokes equations (4.1) become

$$
\left[\frac{\partial}{\partial t}+v k^{2}\right] \tilde{u}_{i}(\boldsymbol{k}, t)=-\frac{\mathrm{i}}{2}\left(\frac{2 \pi}{L}\right)^{3} \tilde{P}_{i j m}(\boldsymbol{k}) \sum_{\substack{\boldsymbol{p} \\(\boldsymbol{k}+\boldsymbol{p}+\boldsymbol{q}=0)}} \sum_{\substack{\boldsymbol{u} \\ j}}(-\boldsymbol{p}, t) \tilde{u}_{m}(-\boldsymbol{q}, t),
$$

where $\tilde{P}_{i j m}(\boldsymbol{k})=k_{m} \tilde{P}_{i j}(\boldsymbol{k})+k_{j} \tilde{P}_{i m}(\boldsymbol{k})$, and the incompressible projection operator is given by $\tilde{P}_{i j}(\boldsymbol{k})=\delta_{i j}-k_{i} k_{j} / k^{2}$. The scalar advection-diffusion equation (3.18) becomes (3.19) in Fourier space, but here we will use the equivalent symmetric form,

$$
\left[\frac{\partial}{\partial t}+\kappa k^{2}\right] \tilde{\theta}(\boldsymbol{k}, t)=-\mu \tilde{u}_{1}(\boldsymbol{k}, t)-\mathrm{i} k_{j}\left(\frac{2 \pi}{L}\right)^{3} \sum_{\substack{\boldsymbol{p} \\(\boldsymbol{k}+\boldsymbol{p}+\boldsymbol{q}=0)}} \sum_{\substack{\boldsymbol{u} \\ j}} \tilde{u}_{j}(-\boldsymbol{p}, t) \tilde{\theta}(-\boldsymbol{q}, t) .
$$

The evolution equation for the Lagrangian position function (4.4) becomes

$$
\frac{\partial}{\partial t} \tilde{\psi}\left(\boldsymbol{k}, t \mid \boldsymbol{k}^{\prime}, t^{\prime}\right)=-\mathrm{i} k_{j}\left(\frac{2 \pi}{L}\right)^{3} \sum_{\substack{\boldsymbol{p} \\(\boldsymbol{k}+\boldsymbol{p}+\boldsymbol{q}=0)}} \sum_{\boldsymbol{q}} \tilde{u}_{j}(-\boldsymbol{p}, t) \tilde{\psi}\left(-\boldsymbol{q}, t \mid \boldsymbol{k}^{\prime}, t^{\prime}\right),
$$

with initial condition

$$
\tilde{\psi}\left(\boldsymbol{k}, t \mid \boldsymbol{k}^{\prime}, t^{\prime}\right)=\frac{L^{3}}{(2 \pi)^{6}} \delta_{k+\boldsymbol{k}^{\prime}}^{3}
$$

$\dagger$ These are available as a supplement to the online version or from the JFM Editorial Office. Cambridge. 
where $\delta_{\boldsymbol{k}+\boldsymbol{k}^{\prime}}^{3}=1$ if $\boldsymbol{k}=-\boldsymbol{k}^{\prime}$, and $\delta_{\boldsymbol{k}+\boldsymbol{k}^{\prime}}^{3}=0$ otherwise. The Fourier transforms of the Lagrangian velocity and scalar fields evolve according to

$$
\begin{aligned}
\frac{\partial}{\partial t} \tilde{v}_{i}\left(t \mid \boldsymbol{k}, t^{\prime}\right)= & -\frac{(2 \pi)^{6}}{L^{3}} v \sum_{\boldsymbol{p}} p^{2} \tilde{u}_{i}(\boldsymbol{p}, t) \tilde{\psi}\left(-\boldsymbol{p}, t \mid \boldsymbol{k}, t^{\prime}\right) \\
& -\mathrm{i} \frac{(2 \pi)^{9}}{L^{6}} \sum_{\substack{\boldsymbol{p} \\
(\boldsymbol{p}+\boldsymbol{q}+\boldsymbol{r}=0)}} \sum_{\boldsymbol{r}} \sum_{r} \frac{r_{i} r_{m} r_{n}}{r^{2}} \tilde{u}_{m}(\boldsymbol{p}, t) \tilde{u}_{n}(\boldsymbol{q}, t) \tilde{\psi}\left(\boldsymbol{r}, t \mid \boldsymbol{k}, t^{\prime}\right),
\end{aligned}
$$

and

$$
\frac{\partial}{\partial t} \tilde{\theta}^{(L)}\left(t \mid \boldsymbol{k}, t^{\prime}\right)=-\mu \tilde{v}_{1}\left(t \mid \boldsymbol{k}, t^{\prime}\right)-\frac{(2 \pi)^{6}}{L^{3}} \kappa \sum_{\boldsymbol{p}} p^{2} \tilde{\theta}(\boldsymbol{p}, t) \tilde{\psi}\left(-\boldsymbol{p}, t \mid \boldsymbol{k}, t^{\prime}\right) .
$$

This may be seen by taking a time derivative of the Fourier space counterparts of (4.6) and (4.7),

$$
\tilde{v}_{i}\left(t \mid \boldsymbol{k}, t^{\prime}\right)=\frac{(2 \pi)^{6}}{L^{3}} \sum_{\boldsymbol{k}^{\prime}} \tilde{u}_{i}\left(\boldsymbol{k}^{\prime}, t\right) \tilde{\psi}\left(-\boldsymbol{k}^{\prime}, t \mid \boldsymbol{k}, t^{\prime}\right),
$$

and

$$
\tilde{\theta}^{(L)}\left(t \mid \boldsymbol{k}, t^{\prime}\right)=\frac{(2 \pi)^{6}}{L^{3}} \sum_{\boldsymbol{k}^{\prime}} \tilde{\theta}\left(\boldsymbol{k}^{\prime}, t\right) \tilde{\psi}\left(-\boldsymbol{k}^{\prime}, t \mid \boldsymbol{k}, t^{\prime}\right) .
$$

The diffusive term in (B 6) is written incorrectly in the paper by Goto \& Kida (1999), although this makes no difference after the SDIP approximations are made.

\section{B.2. Two-point statistics}

We will need evolution equations for the two-point quantities $\tilde{W}_{i}\left(\boldsymbol{k}, t, t^{\prime}\right)$ and $\tilde{Y}_{i}\left(\boldsymbol{k}, t, t^{\prime}\right)$, defined by (4.12) and (4.13), respectively. For one-time correlations we have

$$
\begin{aligned}
& {\left[\frac{\partial}{\partial t}+(\nu+\kappa) k^{2}\right] \tilde{W}_{i}(\boldsymbol{k}, t, t)=-\mu \tilde{V}_{1 i}(\boldsymbol{k}, t, t)}
\end{aligned}
$$

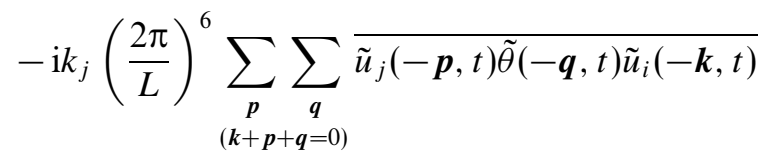

$$
\begin{aligned}
& +\frac{\mathrm{i}}{2}\left(\frac{2 \pi}{L}\right)^{6} \tilde{P}_{i j m}(\boldsymbol{k}) \sum_{\substack{\boldsymbol{p} \\
(-\boldsymbol{k}+\boldsymbol{p}+\boldsymbol{q}=0)}} \sum_{\tilde{u}_{j}(-\boldsymbol{p}, t) \tilde{u}_{m}(-\boldsymbol{q}, t) \tilde{\theta}(\boldsymbol{k}, t)},
\end{aligned}
$$

and $\tilde{Y}_{i}(\boldsymbol{k}, t, t)=\tilde{W}_{i}(-\boldsymbol{k}, t, t)$. For two-time correlations we have that

$$
\begin{aligned}
\frac{\partial}{\partial t} \tilde{W}_{i}\left(\boldsymbol{k}, t, t^{\prime}\right)= & -\mu \tilde{V}_{1 i}\left(\boldsymbol{k}, t, t^{\prime}\right)-\kappa \frac{(2 \pi)^{9}}{L^{6}} \sum_{\boldsymbol{p}} p^{2} \overline{\tilde{\theta}(\boldsymbol{p}, t) \tilde{\psi}\left(-\boldsymbol{p}, t \mid \boldsymbol{k}, t^{\prime}\right) \tilde{u}_{i}\left(-\boldsymbol{k}, t^{\prime}\right)} \\
\frac{\partial}{\partial t} \tilde{Y}_{i}\left(\boldsymbol{k}, t, t^{\prime}\right)= & -\mathrm{i} \frac{(2 \pi)^{12}}{L^{9}} \sum_{\boldsymbol{p}} \sum_{\boldsymbol{q}} \sum_{\boldsymbol{r}} \frac{r_{i} r_{m} r_{n}}{r^{2}} \overline{\left.\tilde{u}_{m}(\boldsymbol{p}, t) \tilde{u}_{n}(\boldsymbol{q}, t) \tilde{\psi}=0\right)}\left(\boldsymbol{r}, t \mid \boldsymbol{k}, t^{\prime}\right) \tilde{\theta}\left(-\boldsymbol{k}, t^{\prime}\right) \\
& -v \frac{(2 \pi)^{9}}{L^{6}} \sum_{\boldsymbol{p}} p^{2} \overline{\tilde{u}_{i}(\boldsymbol{p}, t) \tilde{\psi}\left(-\boldsymbol{p}, t \mid \boldsymbol{k}, t^{\prime}\right) \tilde{\theta}\left(-\boldsymbol{k}, t^{\prime}\right) .}
\end{aligned}
$$


It will be necessary to make use of linear response functions in the SDIP calculation. The Eulerian and Lagrangian scalar response functions are defined as

$$
\begin{aligned}
\tilde{G}\left(\boldsymbol{k}, t \mid \boldsymbol{k}^{\prime}, t^{\prime}\right) & =\frac{\delta \tilde{\theta}(\boldsymbol{k}, t)}{\delta \tilde{\theta}\left(\boldsymbol{k}^{\prime}, t^{\prime}\right)} \\
\tilde{G}^{(L)}\left(t \mid \boldsymbol{k}, \boldsymbol{k}^{\prime}, t^{\prime}\right) & =\frac{\delta \tilde{\theta}(L)\left(t \mid \boldsymbol{k}, t^{\prime}\right)}{\delta \tilde{\theta}\left(\boldsymbol{k}^{\prime}, t^{\prime}\right)},
\end{aligned}
$$

with evolution equations

$$
\begin{aligned}
{\left[\frac{\partial}{\partial t}+\kappa k^{2}\right] \tilde{G}\left(\boldsymbol{k}, t \mid \boldsymbol{k}^{\prime}, t^{\prime}\right) } & =-\mathrm{i} k_{j}\left(\frac{2 \pi}{L}\right)^{3} \sum_{\substack{p \\
(k+p+q=0)}} \sum_{\substack{q \\
j}}(-\boldsymbol{p}, t) \tilde{G}\left(-\boldsymbol{q}, t \mid \boldsymbol{k}^{\prime}, t^{\prime}\right), \\
\frac{\partial}{\partial t} \tilde{G}^{(L)}\left(t \mid \boldsymbol{k}, \boldsymbol{k}^{\prime}, t^{\prime}\right) & =-\kappa \frac{(2 \pi)^{6}}{L^{3}} \sum_{p} p^{2} \tilde{G}\left(\boldsymbol{p}, t \mid \boldsymbol{k}^{\prime}, t^{\prime}\right) \tilde{\psi}\left(-\boldsymbol{p}, t \mid \boldsymbol{k}, t^{\prime}\right),
\end{aligned}
$$

and initial conditions

$$
\tilde{G}\left(\boldsymbol{k}, t^{\prime} \mid \boldsymbol{k}^{\prime}, t^{\prime}\right)=\tilde{G}^{(L)}\left(t^{\prime} \mid \boldsymbol{k}, \boldsymbol{k}^{\prime}, t^{\prime}\right)=\frac{L^{3}}{(2 \pi)^{6}} \delta_{k+k^{\prime}}^{3} .
$$

Here $\delta$ is a functional derivative, and our notation is consistent so that, for example, $\delta \tilde{\theta}(\boldsymbol{k}, t) / \delta \tilde{\theta}\left(\boldsymbol{k}^{\prime}, t^{\prime}\right)$ is a Fourier transform with respect to $\boldsymbol{x}$, followed by a Fourier transform with respect to $\boldsymbol{x}^{\prime}$ of $\delta \theta(\boldsymbol{x}, t) / \delta \theta\left(\boldsymbol{x}^{\prime}, t^{\prime}\right)$. Similarly, we define Eulerian and Lagrangian velocity response functions as

$$
\begin{aligned}
\tilde{G}_{i j}^{(E)}\left(\boldsymbol{k}, t \mid \boldsymbol{k}^{\prime}, t^{\prime}\right) & =\frac{\delta \tilde{u}_{i}(\boldsymbol{k}, t)}{\delta \tilde{u}_{j}\left(\boldsymbol{k}^{\prime}, t^{\prime}\right)}, \\
\tilde{G}_{i j}^{(L)}\left(t \mid \boldsymbol{k}, \boldsymbol{k}^{\prime}, t^{\prime}\right) & =\frac{\delta \tilde{v}_{i}\left(t \mid \boldsymbol{k}, t^{\prime}\right)}{\delta \tilde{u}_{j}\left(\boldsymbol{k}^{\prime}, t^{\prime}\right)},
\end{aligned}
$$

with initial conditions

$$
\tilde{G}_{i j}^{(E)}\left(\boldsymbol{k}, t^{\prime} \mid \boldsymbol{k}^{\prime}, t^{\prime}\right)=\tilde{G}_{i j}^{(L)}\left(t^{\prime} \mid \boldsymbol{k}, \boldsymbol{k}^{\prime}, t^{\prime}\right)=\frac{L^{3}}{(2 \pi)^{6}} \delta_{i j} \delta_{\boldsymbol{k}+\boldsymbol{k}^{\prime}}^{3}
$$

Details of the evolution equations for $\tilde{G}_{i j}^{(E)}\left(\boldsymbol{k}, t \mid \boldsymbol{k}^{\prime}, t^{\prime}\right)$ and $\tilde{G}_{i j}^{(L)}\left(t \mid \boldsymbol{k}, \boldsymbol{k}^{\prime}, t^{\prime}\right)$ may be found in $\mathrm{KG}$.

We will mostly work with the incompressible projection of $\tilde{G}_{i j}^{(L)}\left(t \mid \boldsymbol{k}, \boldsymbol{k}^{\prime}, t^{\prime}\right)$, and so we define

$$
\tilde{G}_{i j}\left(\boldsymbol{k}, t, t^{\prime}\right)=\frac{(2 \pi)^{6}}{L^{3}} \overline{\tilde{G}_{i m}^{(L)}\left(t \mid \boldsymbol{k},-\boldsymbol{k}, t^{\prime}\right)} \tilde{P}_{m j}(\boldsymbol{k}) .
$$

\section{B.3. DIA decompositions}

The basis of the SDIP is the decomposition of the field variables into the sum of non-direct-interaction (NDI) fields, denoted by the superscript (0), and the deviation fields, denoted with the superscript (1). For example, we decompose the scalar field as

$$
\tilde{\theta}(\boldsymbol{k}, t)=\tilde{\theta}^{(0)}\left(\boldsymbol{k}, t \| \boldsymbol{k}_{0}, \boldsymbol{p}_{0}, \boldsymbol{q}_{0}\right)+\tilde{\theta}^{(1)}\left(\boldsymbol{k}, t \| \boldsymbol{k}_{0}, \boldsymbol{p}_{0}, \boldsymbol{q}_{0}\right),
$$

where $\boldsymbol{k}_{0}, \boldsymbol{p}_{0}$ and $\boldsymbol{q}_{0}$ are a triad of wavevectors such that $\boldsymbol{k}_{0}+\boldsymbol{p}_{0}+\boldsymbol{q}_{0}=0$. The initial conditions for this decomposition are given at time $t_{0}$ as

$$
\tilde{\theta}^{(0)}\left(\boldsymbol{k}, t_{0} \| \boldsymbol{k}_{0}, \boldsymbol{p}_{0}, \boldsymbol{q}_{0}\right)=\tilde{\theta}\left(\boldsymbol{k}, t_{0}\right), \quad \tilde{\theta}^{(1)}\left(\boldsymbol{k}, t_{0} \| \boldsymbol{k}_{0}, \boldsymbol{p}_{0}, \boldsymbol{q}_{0}\right)=0 .
$$


The evolution of $\tilde{\theta}^{(0)}$ is governed by

$$
\begin{aligned}
{\left[\frac{\partial}{\partial t}+\kappa k^{2}\right] \tilde{\theta}^{(0)}\left(\boldsymbol{k}, t \| \boldsymbol{k}_{0}, \boldsymbol{p}_{0}, \boldsymbol{q}_{0}\right)=} & -\mathrm{i} k_{j}\left(\frac{2 \pi}{L}\right)^{3} \sum_{\substack{\boldsymbol{p} \\
(\boldsymbol{k}+\boldsymbol{p}+\boldsymbol{q}=0)}}^{\prime} \sum_{\boldsymbol{q}} \tilde{u}_{j}(-\boldsymbol{p}, t) \tilde{\theta}^{(0)}\left(-\boldsymbol{q}, t \| \boldsymbol{k}_{0}, \boldsymbol{p}_{0}, \boldsymbol{q}_{0}\right) \\
& -\mu \tilde{u}_{1}(\boldsymbol{k}, t),
\end{aligned}
$$

where $\sum \sum^{\prime}$ denotes a summation that excludes interactions between the triad $\boldsymbol{k}_{0}$, $\boldsymbol{p}_{0}$, and $\boldsymbol{q}_{0}$. Subtracting (B 23) from (B 2) we find

$$
\begin{aligned}
{\left[\frac{\partial}{\partial t}+\kappa k^{2}\right] \tilde{\theta}^{(1)}\left(\boldsymbol{k}, t \| \boldsymbol{k}_{0}, \boldsymbol{p}_{0}, \boldsymbol{q}_{0}\right)=} & -\mathrm{i} k_{j}\left(\frac{2 \pi}{L}\right)^{3} \sum_{\substack{\left.\boldsymbol{p} \\
\boldsymbol{k}_{+}+\boldsymbol{p}+\boldsymbol{q}=0\right)}}^{\prime} \tilde{u}_{j}(-\boldsymbol{p}, t) \tilde{\theta}^{(1)}\left(-\boldsymbol{q}, t \| \boldsymbol{k}_{0}, \boldsymbol{p}_{0}, \boldsymbol{q}_{0}\right) \\
& -\mathrm{i} \delta_{\boldsymbol{k}-\boldsymbol{k}_{0}}^{3} k_{0 j} \tilde{u}_{j}\left(-\boldsymbol{p}_{0}, t\right) \tilde{\theta}^{(0)}\left(-\boldsymbol{q}_{0}, t \| \boldsymbol{k}_{0}, \boldsymbol{p}_{0}, \boldsymbol{q}_{0}\right) \\
& -\mathrm{i} \delta_{\boldsymbol{k}-\boldsymbol{k}_{0}}^{3} k_{0 j} \tilde{u}_{j}\left(-\boldsymbol{q}_{0}, t\right) \tilde{\theta}^{(0)}\left(-\boldsymbol{p}_{0}, t \| \boldsymbol{k}_{0}, \boldsymbol{p}_{0}, \boldsymbol{q}_{0}\right) \\
& +\mathrm{i} \delta_{\boldsymbol{k}+\boldsymbol{k}_{0}}^{3} k_{0 j} \tilde{u}_{j}\left(\boldsymbol{p}_{0}, t\right) \tilde{\theta}^{(0)}\left(\boldsymbol{q}_{0}, t \| \boldsymbol{k}_{0}, \boldsymbol{p}_{0}, \boldsymbol{q}_{0}\right) \\
& +\mathrm{i} \delta_{\boldsymbol{k}+\boldsymbol{k}_{0}}^{3} k_{0 j} \tilde{u}_{j}\left(\boldsymbol{q}_{0}, t\right) \tilde{\theta}^{(0)}\left(\boldsymbol{p}_{0}, t \| \boldsymbol{k}_{0}, \boldsymbol{p}_{0}, \boldsymbol{q}_{0}\right) \\
& +\left(\boldsymbol{k}_{0} \rightarrow \boldsymbol{p}_{0} \rightarrow \boldsymbol{q}_{0} \rightarrow \boldsymbol{k}_{0}\right) .
\end{aligned}
$$

Similar decompositions are made for the Eulerian velocity field, $\tilde{u}(\boldsymbol{k}, t)$, the position function, $\tilde{\psi}\left(\boldsymbol{k}, t \mid \boldsymbol{k}^{\prime}, t^{\prime}\right)$, the Eulerian velocity response function $\tilde{G}_{i j}^{(E)}\left(\boldsymbol{k}, t \mid \boldsymbol{k}^{\prime}, t^{\prime}\right)$, and the Lagrangian velocity response function $\tilde{G}_{i j}^{(L)}\left(t \mid \boldsymbol{k}, \boldsymbol{k}^{\prime}, t^{\prime}\right)$, see KG. We also decompose the Eulerian scalar response function, $\tilde{G}\left(\boldsymbol{k}, t \mid \boldsymbol{k}^{\prime}, \boldsymbol{t}^{\prime}\right)$, see Goto \& Kida (1999). The deviation fields can then be expressed in terms of the NDI fields and the response functions. For example, the scalar deviation field is given by

$$
\begin{aligned}
\tilde{\theta}^{(1)}\left(\boldsymbol{k}, t \| \boldsymbol{k}_{0}, \boldsymbol{p}_{0}, \boldsymbol{q}_{0}\right)= & -\mathrm{i} k_{j} \frac{(2 \pi)^{9}}{L^{6}} \int_{t_{0}}^{t} \mathrm{~d} t^{\prime} \tilde{G}^{(0)}\left(\boldsymbol{k}, t \mid-\boldsymbol{k}, t^{\prime} \| \boldsymbol{k}_{0}, \boldsymbol{p}_{0}, \boldsymbol{q}_{0}\right) \\
& \times\left[\delta_{\boldsymbol{k} \boldsymbol{k}_{0}}^{3} \tilde{u}_{j}\left(-\boldsymbol{p}_{0}, t^{\prime}\right) \tilde{\theta}^{(0)}\left(-\boldsymbol{q}_{0}, t^{\prime} \| \boldsymbol{k}_{0}, \boldsymbol{p}_{0}, \boldsymbol{q}_{0}\right)\right. \\
& +\delta_{\boldsymbol{k}-\boldsymbol{k}_{0}}^{3} \tilde{u}_{j}\left(-\boldsymbol{q}_{0}, t^{\prime}\right) \tilde{\theta}^{(0)}\left(-\boldsymbol{p}_{0}, t^{\prime} \| \boldsymbol{k}_{0}, \boldsymbol{p}_{0}, \boldsymbol{q}_{0}\right) \\
& +\delta_{\boldsymbol{k}+\boldsymbol{k}_{0}}^{3} \tilde{u}_{j}\left(\boldsymbol{p}_{0}, t^{\prime}\right) \tilde{\theta}^{(0)}\left(\boldsymbol{q}_{0}, t^{\prime} \| \boldsymbol{k}_{0}, \boldsymbol{p}_{0}, \boldsymbol{q}_{0}\right) \\
& +\delta_{\boldsymbol{k}+\boldsymbol{k}_{0}}^{3} \tilde{u}_{j}\left(\boldsymbol{q}_{0}, t^{\prime}\right) \tilde{\theta}^{(0)}\left(\boldsymbol{p}_{0}, t^{\prime} \| \boldsymbol{k}_{0}, \boldsymbol{p}_{0}, \boldsymbol{q}_{0}\right) \\
& \left.+\left(\boldsymbol{k}_{0} \rightarrow \boldsymbol{p}_{0} \rightarrow \boldsymbol{q}_{0} \rightarrow \boldsymbol{k}_{0}\right)\right] .
\end{aligned}
$$

B.4. Evolution equations for $\tilde{W}(\boldsymbol{k}, t, t), \tilde{W}\left(\boldsymbol{k}, t, t^{\prime}\right)$ and $\tilde{X}\left(\boldsymbol{k}, t, t^{\prime}\right)$

The main purpose of the SDIP is to express third-order correlations in terms of second-order correlations so that closed evolution equations for second-order quantities can be derived. There are three main assumptions in the SDIP procedure: (i) The magnitude of the deviation field is smaller than that of the NDI field for times $\left(t-t_{0}\right)$ within the correlation time scale of the velocity field. (ii) Any two Fourier modes of the NDI fields without direct interaction are statistically independent of each other. For example, $\tilde{\theta}^{(0)}\left(\boldsymbol{k}_{0}, t \| \boldsymbol{k}_{0}, \boldsymbol{p}_{0}, \boldsymbol{q}_{0}\right), \tilde{\theta}^{(0)}\left(\boldsymbol{p}_{0}, t^{\prime} \| \boldsymbol{k}_{0}, \boldsymbol{p}_{0}, \boldsymbol{q}_{0}\right)$ and $\tilde{u}_{k}^{(0)}\left(\boldsymbol{q}_{0}, t^{\prime \prime} \| \boldsymbol{k}_{0}, \boldsymbol{p}_{0}, \boldsymbol{q}_{0}\right)$ are statistically independent. (iii) The NDI position function field, $\tilde{\psi}^{(0)}$, is statistically independent of the other Eulerian quantities, such as $\tilde{u}_{i}^{(0)}$ and $\tilde{\theta}^{(0)}$. Additional statistical assumptions were required in $\mathrm{KG}$ involving the position response function, but this function is not used here. 
Assumptions (i) and (ii) were tested for a model system in Goto \& Kida (1998, 2002), but assumption (iii) is difficult to justify. Assumption (iii) will be used several times throughout the derivation to reduce Lagrangian averages, expressed using the position function, to simpler Eulerian averages. It could be argued that this crude treatment of the statistics of the position function means that the SDIP is not a truly Lagrangian closure theory. We nonetheless proceed with this assumption as it leads to a tractable closure.

SDIP approximations to (B 9) and (B 10) are derived in Appendix C using the DIA decompositions and the above three assumptions, and following the method introduced by KG and Goto \& Kida (1999). The results are

$$
\begin{aligned}
{\left[\frac{\partial}{\partial t}+(\nu+\kappa) k^{2}\right] \tilde{W}_{i}(\boldsymbol{k}, t, t)=} & \left(\frac{2 \pi}{L}\right)^{3} \sum_{\substack{p \\
(\boldsymbol{k}+\boldsymbol{p}+\boldsymbol{q}=0)}} \sum_{t_{0}} \int_{t_{0}}^{t} \mathrm{~d} t^{\prime} L_{i}\left(\boldsymbol{k}, \boldsymbol{p}, \boldsymbol{q}, t, t^{\prime}\right) \\
& -\mu \tilde{V}_{1 i}(\boldsymbol{k}, t, t),
\end{aligned}
$$

where

$$
\begin{aligned}
& L_{i}\left(\boldsymbol{k}, \boldsymbol{p}, \boldsymbol{q}, t, t^{\prime}\right) \\
&= k_{j}\left(\tilde{Q}_{i b}\left(-\boldsymbol{k}, t, t^{\prime}\right) \tilde{W}_{c}\left(-\boldsymbol{q}, t, t^{\prime}\right)\left[p_{c} \tilde{G}_{j b}\left(-\boldsymbol{p}, t, t^{\prime}\right)+p_{b} \tilde{G}_{j c}\left(-\boldsymbol{p}, t, t^{\prime}\right)\right]\right. \\
&+(\mathrm{i} \leftrightarrow j, \boldsymbol{k} \leftrightarrow \boldsymbol{p}))+k_{j} q_{l} \exp \left[-\kappa q^{2}\left(t-t^{\prime}\right)\right]\left[\tilde{Q}_{j l}\left(-\boldsymbol{p}, t, t^{\prime}\right) \tilde{X}_{i}\left(-\boldsymbol{k}, t, t^{\prime}\right)\right. \\
&\left.+\tilde{Q}_{i l}\left(-\boldsymbol{k}, t, t^{\prime}\right) \tilde{X}_{j}\left(-\boldsymbol{p}, t, t^{\prime}\right)\right]+\tilde{P}_{i j m}(\boldsymbol{k})\left(\tilde { Q } _ { m c } ( \boldsymbol { q } , t , t ^ { \prime } ) \tilde { W } _ { b } ( \boldsymbol { k } , t , t ^ { \prime } ) \left[p_{c} \tilde{G}_{j b}\left(\boldsymbol{p}, t, t^{\prime}\right)\right.\right. \\
&\left.+p_{b} \tilde{G}_{j c}\left(\boldsymbol{p}, t, t^{\prime}\right)\right]+\frac{1}{2} k_{l} \tilde{P}_{i j m}(\boldsymbol{k}) \exp \left[-\kappa k^{2}\left(t-t^{\prime}\right)\right]\left[\tilde{Q}_{j l}\left(\boldsymbol{p}, t, t^{\prime}\right) \tilde{X}_{m}\left(\boldsymbol{q}, t, t^{\prime}\right)\right. \\
&\left.+\tilde{Q}_{m l}\left(\boldsymbol{q}, t, t^{\prime}\right) \tilde{X}_{j}\left(\boldsymbol{p}, t, t^{\prime}\right)\right],
\end{aligned}
$$

and

$$
\left[\frac{\partial}{\partial t}+\kappa k^{2}\right] \tilde{W}_{i}\left(\boldsymbol{k}, t, t^{\prime}\right)=-\mu \tilde{V}_{1 i}\left(\boldsymbol{k}, t, t^{\prime}\right) .
$$

It is easier to work with $\tilde{X}_{i}\left(\boldsymbol{k}, t, t^{\prime}\right)$, defined by (4.15), rather than $\tilde{Y}_{i}\left(\boldsymbol{k}, t, t^{\prime}\right)$, and so the SDIP approximation to the incompressible projection of (B 11) is derived in Appendix D,

$$
\begin{aligned}
& {\left[\frac{\partial}{\partial t}+\nu k^{2}\right] \tilde{X}_{i}\left(\boldsymbol{k}, t, t^{\prime}\right)} \\
& \quad=-2\left(\frac{2 \pi}{L}\right)^{3} \sum_{\substack{p \\
(\boldsymbol{k}+\boldsymbol{p}+\boldsymbol{q}=0)}} \sum_{q} \tilde{P}_{i l}(\boldsymbol{k}) \frac{q_{l} q_{m} q_{n} q_{j}}{q^{2}} \tilde{X}_{n}\left(\boldsymbol{k}, t, t^{\prime}\right) \int_{t^{\prime}}^{t} \mathrm{~d} t^{\prime \prime} \tilde{Q}_{m j}\left(\boldsymbol{p}, t, t^{\prime \prime}\right) .
\end{aligned}
$$

Taking the $L \rightarrow \infty$ limit, the system of integro-differential equations to be solved can be summarized as

$$
\begin{aligned}
& {\left[\frac{\partial}{\partial t}+(v+\kappa) k^{2}\right] \tilde{W}_{i}(\boldsymbol{k}, t, t)=\int \mathrm{d} \boldsymbol{p} \int \mathrm{d} \boldsymbol{q} \delta_{\boldsymbol{k}+\boldsymbol{p}+\boldsymbol{q}}^{3} \int_{t_{0}}^{t} \mathrm{~d} t^{\prime} L_{i}\left(\boldsymbol{k}, \boldsymbol{p}, \boldsymbol{q}, t, t^{\prime}\right)} \\
& -\mu \tilde{V}_{1 i}(\boldsymbol{k}, t, t), \\
& {\left[\frac{\partial}{\partial t}+v k^{2}\right] \tilde{X}_{i}\left(\boldsymbol{k}, t, t^{\prime}\right)} \\
& =-2 \tilde{P}_{i l}(\boldsymbol{k}) \int \mathrm{d} \boldsymbol{p} \int \mathrm{d} \boldsymbol{q} \delta_{\boldsymbol{k}+\boldsymbol{p}+\boldsymbol{q}}^{3} \frac{q_{l} q_{m} q_{n} q_{j}}{q^{2}} \tilde{X}_{n}\left(\boldsymbol{k}, t, t^{\prime}\right) \int_{t^{\prime}}^{t} \mathrm{~d} t^{\prime \prime} \tilde{Q}_{m j}\left(\boldsymbol{p}, t, t^{\prime \prime}\right),
\end{aligned}
$$


together with (B 28) for $\tilde{W}_{i}\left(\boldsymbol{k}, t, t^{\prime}\right)$ and the initial condition,

$$
\tilde{X}_{i}(\boldsymbol{k}, t, t)=\tilde{W}_{i}(-\boldsymbol{k}, t, t) .
$$

This is a closed system of equations for $\tilde{W}_{i}\left(\boldsymbol{k}, t, t^{\prime}\right)$ and $\tilde{X}_{i}\left(\boldsymbol{k}, t, t^{\prime}\right)$ once the velocity field statistics $\tilde{V}_{i j}\left(\boldsymbol{k}, t, t^{\prime}\right), \tilde{Q}_{i j}\left(\boldsymbol{k}, t, t^{\prime}\right)$ and $\tilde{G}_{i j}\left(\boldsymbol{k}, t, t^{\prime}\right)$ are specified.

\section{B.5. Spatial symmetries and stationarity}

We now use the spatial symmetries and stationarity of the problem to simplify our equations. The velocity field is isotropic and stationary, and so we can write (4.16) and

$$
\tilde{G}_{i j}\left(\boldsymbol{k}, t, t^{\prime}\right)=\tilde{P}_{i j}(\boldsymbol{k}) G^{\dagger}\left(k, t-t^{\prime}\right) .
$$

Note that although $\tilde{G}_{i j}\left(\boldsymbol{k}, t, t^{\prime}\right)$ does not satisfy $k_{i} \tilde{G}_{i j}\left(\boldsymbol{k}, t, t^{\prime}\right)=0$ in the general case, the incompressible property $k_{j} \tilde{G}_{i j}\left(\boldsymbol{k}, t, t^{\prime}\right)=0$ is sufficient to give the form (B 33) in the isotropic case. Similarly, the condition $k_{j} \tilde{V}_{i j}\left(\boldsymbol{k}, t, t^{\prime}\right)=0$ together with isotropy is sufficient to ensure that

$$
\tilde{V}_{i j}\left(\boldsymbol{k}, t, t^{\prime}\right)=\frac{1}{2} \tilde{P}_{i j}(\boldsymbol{k}) V^{\dagger}\left(k, t-t^{\prime}\right) .
$$

The definition (4.1.4) of $\tilde{Q}_{i j}\left(\boldsymbol{k}, t, t^{\prime}\right)$ then implies that $V^{\dagger}(k, t)=Q^{\dagger}(k, t)$.

We turn now to statistical quantities involving the scalar. It will be convenient to generalize briefly to the case of an arbitrary mean scalar gradient $\mu_{i}$. Axisymmetry and the condition $k_{i} \tilde{W}_{i}\left(\boldsymbol{k}, t, t^{\prime}\right)=0$ imply that,

$$
\tilde{W}_{i}\left(\boldsymbol{k}, t, t^{\prime}\right)=f\left(k, t, t^{\prime}, \boldsymbol{\mu}, k_{j} \boldsymbol{\mu}_{j}\right)\left(\mu_{i}-k_{i} \frac{k_{s} \mu_{s}}{k^{2}}\right) .
$$

The scalar, and therefore $\tilde{W}_{i}\left(\boldsymbol{k}, t, t^{\prime}\right)$, depend linearly on the mean scalar gradient $\mu_{i}$ after initial fluctuations decay, and so we can write $\tilde{W}_{i}\left(\boldsymbol{k}, t, t^{\prime}\right)=\tilde{P}_{i j}(\boldsymbol{k}) \mu_{j} W^{\dagger}\left(k, t-t^{\prime}\right)$. In our case, the mean scalar gradient is in the ' 1 ' direction and so we find (4.17). Using a similar argument for the form of $\tilde{X}_{i}\left(\boldsymbol{k}, t, t^{\prime}\right)$, we find

$$
\tilde{X}_{i}\left(\boldsymbol{k}, t, t^{\prime}\right)=\tilde{P}_{i 1}(\boldsymbol{k}) \mu X^{\dagger}\left(k, t-t^{\prime}\right) .
$$

Substituting into (B 28) leads to

$$
\left[\frac{\partial}{\partial t}+\kappa k^{2}\right] W^{\dagger}(k, t)=-\frac{1}{2} Q^{\dagger}(k, t),
$$

and this may be solved to give (4.18).

Making a comparison between the evolution equation for $\tilde{Q}_{i j}\left(\boldsymbol{k}, t, t^{\prime}\right)$ in $\mathrm{KG}$, and the evolution equation (B 29) for $\tilde{X}_{i}\left(\boldsymbol{k}, t, t^{\prime}\right)$ here, it is easy to show that $X^{\dagger}(k, t)$ and $Q^{\dagger}(k, t)$ have the same evolution equation. This can be written as

$$
\left[\frac{\partial}{\partial t}+v k^{2}+\hat{\eta}(k, t)\right] X^{\dagger}(k, t)=0
$$

where

$$
\hat{\eta}(k, t)=\frac{4 \pi}{3} k^{5} \int_{0}^{\infty} \mathrm{d} p p^{10 / 3} J\left(p^{2 / 3}\right) \int_{0}^{t} \mathrm{~d} t^{\prime} Q^{\dagger}\left(k p, t^{\prime}\right),
$$

and

$$
J(p)=\frac{3}{32 p^{5}}\left(\frac{\left(1-p^{3}\right)^{4}}{2 p^{3 / 2}} \log \left[\frac{1+p^{3 / 2}}{\left|1-p^{3 / 2}\right|}\right]-\frac{1+p^{3}}{3}\left(3 p^{6}-14 p^{3}+3\right)\right)
$$


Therefore,

$$
X^{\dagger}(k, t)=\frac{Q^{\dagger}(k, t)}{Q^{\dagger}(k, 0)} W^{\dagger}(k, 0),
$$

where we have used (B 32). Finally, we substitute the isotropic forms into the integro-differential equation (B 30) for $\tilde{W}_{i}(\boldsymbol{k}, t, t)$. The calculation is made easier by recasting (B 30) in the form $\mathscr{L}_{i j} \mu_{j}=0$, for a general scalar gradient $\mu_{j}$, and then proceeding by setting $\mathscr{L}_{i i}=0$. Using (B 41), and the important relation derived in $\mathrm{KG}, Q^{\dagger}(k, t)=G^{\dagger}(k, t) Q^{\dagger}(k, 0)$, and after considerable algebra, we find the integral equation (4.19). Note that we have let $\left(t-t_{0}\right) \rightarrow \infty$, and this is justified by the exponential decay of $Q^{\dagger}(k, t)$ with respect to $t$. Extensive use has been made of relations between geometric factors such as $k^{2} \sigma(k, p, q)=k_{j} k_{m} \tilde{P}_{j m}(\boldsymbol{p})$.

\section{REFERENCES}

BATCHELOR, G. K. 1959 Small-scale variation of convected quantities like temperature in turbulent fluid. Part 1. General discussion and the case of small conductivity. J. Fluid Mech. 5, 113-133.

Batchelor, G. K., Howells, I. D. \& Townsend, A. A. 1959 Small-scale variation of convected quantities like temperature in turbulent fluid. Part 2. The case of large conductivity. J. Fluid Mech. 5, 134-139.

Bendat, J. \& Piersol, A. 1986 Random Data - Analysis and Measurement Procedures, 2nd edn. Wiley.

Bos, W. J. T., Touil, H., Shao, L. \& Bertoglio, J. P. 2004 On the behaviour of the velocity-scalar cross-correlation spectrum in the inertial range. Phys. Fluids 16, 3818-3823.

Chasnov, J. R. 1991 Simulation of the inertial-conductive subrange. Phys. Fluids A 3, 1164-1168.

Dimotakis, P. E. \& Miller, P. L. 1990 Some consequences of the boundedness of scalar fluctuations. Phys. Fluids A 2, 1919-1920.

Galassi, M., Davies, J., Theiler, J., Gough, B., Jungman, G., Booth, M. \& Rossi, F. 2001 GNU Scientific Library Reference Manual - 2nd. edn. Network Theory.

Gargett, A. E., Merryfield, W. J. \& Holloway, G. 2003 Direct numerical simulation of differential scalar diffusion in three-dimensional stratified turbulence. J. Phys. Oceanogr. 33, 1758-1782.

Gibson, C. H. 1968 Fine structure of scalar fields mixed by turbulence II. Spectral theory. Phys. Fluids 11, 2316-2327.

Goto, S. \& KIDA, S. 1998 Direct-interaction approximation and Reynolds-number reversed expansion for a dynamical system. Physica D 117, 191-214.

Goto, S. \& KIDA, S. 1999 Pasive scalar spectrum in isotropic turbulence: prediction by the Lagrangian direct-interaction approximation. Phys. Fluids 11, 1936-1952.

Goto, S. \& KIDA, S. 2002 Sparseness of nonlinear coupling: importance in sparse direct-interaction perturbation. Nonlinearity 15, 1499-1520.

Goтон, T. 1989 Passive scalar diffusion in two-dimensional turbulence in the Lagrangian renormalized approximation. J. Phys. Soc. Japan 58, 2365-2379.

Gotoh, T., NaGaKi, J. \& Kaneda, Y. 2000 Passive scalar spectrum in the viscous-convective range in two-dimensional steady turbulence. Phys. Fluids 12, 155-168.

Herr, S., WANG, L. \& Collins, L. R. 1996 EDQNM model of a passive scalar with a uniform mean gradient. Phys. Fluids 8, 1588-1608.

KANEDA, Y. 1981 Renormalized expansions in the theory of turbulence with the use of the Lagrangian position function. J. Fluid Mech. 107, 131-145.

KANEDA, Y. 1986 Inertial range structure of turbulent velocity and scalar fields in a Lagrangian renormalized approximation. Phys. Fluids 29, 701-708.

Kaneda, Y. \& YoshidA, K. 2004 Small-scale anisotropy in stably stratified turbulence. New J. Phys. 6, article 34.

KIDA, S. \& GoTo, S. 1997 A Lagrangian direct-interaction approximation for homogeneous isotropic turbulence. J. Fluid Mech. 345, 307-345.

Kraichnan, R. H. 1959 The structure of isotropic turbulence at very high Reynolds number. J. Fluid Mech. 5, 497-543. 
Kraichnan, R. H. 1965 Lagrangian-history closure approximation for turbulence. Phys. Fluids 8, $575-598$.

LESLIE, D. 1973 Developments in the Theory of Turbulence. Oxford University Press.

Lumley, J. L. 1967 Similarity and the turbulent energy spectrum. Phys. Fluids 10, 855-858.

Lundgren, T. S. 1982 Strained spiral vortex model for turbulent fine structure. Phys. Fluids 25, 2193-2203.

MсСомв, W. D. 1990 The Physics of Fluid Turbulence. Oxford University Press.

MсСомв, W. D., Filipiak, M. J. \& Shanmugasundaram, V. 1992 Rederivation and further assessment of the LET theory of isotropic turbulence, as applied to passive scalar convection. J. Fluid Mech. 245, 279-300.

Miller, P. L. \& Dimotakis, P. E. 1996 Measurements of scalar power spectra in high Schmidt number turbulent jets. J. Fluid Mech 308, 129-146.

Misra, A. \& Pullin, D. I. 1997 A vortex-based subgrid stress model for large-eddy simulation. Phys. Fluids 9, 2443-2454.

MydlarsKi, L. 2003 Mixed velocity-passive scalar statistics in high-Reynolds-number turbulence. J. Fluid Mech. 475, 173-203.

Mydlarski, L. \& Warhaft, Z. 1998 Passive scalar statistics in high-Péclet-number grid turbulence. J. Fluid Mech. 358, 135-175.

O'Gorman, P. A. \& Pullin, D. I. 2003 The velocity-scalar cross spectrum of stretched spiral vortices. Phys. Fluids 15, 280-291.

O'Gorman, P. A. \& Pullin, D. I. 2004 On modal time correlations of turbulent velocity and scalar fields. J. Turbulence 5, article 35 .

Pullin, D. I. 2000 A vortex-based model for the subgrid flux of a passive scalar. Phys. Fluids 12, 2311-2319.

Shraiman, B. L. \& Siggia, E. D. 2000 Scalar turbulence. Nature 405, 639-646.

SReEnivasan, K. R. 1995 On the universality of the Kolmogorov constant. Phys. Fluids 7, 2778-2784.

Tennekes, H. \& Lumley, J. L. 1974 A First Course in Turbulence. MIT Press.

Voelkl, T., Pullin, D. I. \& Chan, D. C. 2000 A physical-space version of the stretched-vortex subgrid-stress model for large-eddy simulation. Phys. Fluids 12, 1810-1825.

Warhaft, Z. 2000 Passive scalars in turbulent flows. Annu. Rev. Fluid Mech. 32, 203-240.

Yeung, P. K., Xu, S. \& Sreenivasan, K. R. 2002 Schmidt number effects on turbulent transport with uniform mean scalar gradient. Phys. Fluids 14, 4178-4191. 